\title{
8
}

\section{Metal/Nucleic-Acid Interactions}

\author{
JACQUELINE K. BARTON \\ Division of Chemistry and Chemical Engineering \\ California Institute of Technology
}

\section{INTRODUCTION}

The interest of the bioinorganic community in the field of metal/nucleic-acid interactions has burgeoned in the last decade. This interest and the resulting progress have come about primarily because of the tremendous advances that have occurred in nucleic-acid technology. We can now isolate, manipulate, and even synthesize nucleic acids of defined sequence and structure, as we would other molecules that chemists commonly explore. Furthermore, as may be evident already in other chapters of this book, bioinorganic chemistry has itself been evolving from a field focused on delineating metal centers in biology to one that includes also the application of inorganic chemistry to probe biological structures and function. In the past decades it has become clear that nucleic acids, structurally, functionally and even remarkably in terms of catalysis, play active and diverse roles in Nature. Transition-metal chemistry, both in the cell and in the chemist's test tube, provides a valuable tool both to accomplish and to explore these processes.

There are also many practical motivations behind the study of how metal ions and complexes interact with nucleic acids. Heavy-metal toxicity in our environment arises in part from the covalent interactions of heavy-metal ions with nucleic acids. In addition, these heavy metals interfere with metalloregulatory proteins and in so doing disrupt gene expression. We need to understand the functioning of the natural metalloregulators of gene expression and we need to design new metal-specific ligands, which, like the proteins themselves, capture heavy metals before their damage is done. Heavy-metal interactions with nucleic acids indeed have provided the basis also for the successful application of cisplatin and its derivatives as anticancer chemotherapeutic agents (see Chapter 9). The design of new pharmaceuticals like cisplatin requires a detailed understanding of how platinum and other metal ions interact with nucleic acids and nucleic-acid processing. Furthermore, we are finding that metal complexes can be uniquely useful in developing spectroscopic and reactive probes of nu- 
cleic acids, and hence may become valuable in developing new diagnostic agents. Finally, Nature itself takes advantage of metal/nucleic-acid chemistry, from the biosynthesis of natural products such as bleomycin, which chelates redox-active metal ions to target and damage foreign DNA, to the development of basic structural motifs for eukaryotic regulatory proteins, the zinc-finger proteins, which bind to DNA and regulate transcription. In all these endeavors, we need first to develop an understanding of how transition-metal ions and complexes interact with nucleic acids and how this chemistry may best be exploited.

In this chapter we first summarize the "basics" needed to consider the interactions of metal ions and complexes with nucleic acids. What are the structures of nucleic acids? What is the basic repertoire of modes of association and chemical reactions that occur between coordination complexes and polynucleotides? We then consider in some detail the interaction of a simple family of coordination complexes, the tris(phenanthroline) metal complexes, with DNA and RNA to illustrate the techniques, questions, and applications of metal/nucleic-acid chemistry that are currently being explored. In this section, the focus on tris(phenanthroline) complexes serves as a springboard to compare and contrast studies of other, more intricately designed transition-metal complexes (in the next section) with nucleic acids. Last we consider how Nature uses metal ions and complexes in carrying out nucleic-acid chemistry. Here the principles, techniques, and fundamental coordination chemistry of metals with nucleic acids provide the foundation for our current understanding of how these fascinating and complex bioinorganic systems may function.

\section{THE BASICS}

\section{A. Nucleic-Acid Structures ${ }^{1}$}

Figure 8.1 displays a single deoxyribonucleotide and the four different nucleicacid bases. As may be evident, each mononucleotide along a nucleic-acid polymer contains a variety of sites for interactions with metal ions, from electrostatic interactions with the anionic phosphate backbone to soft nucleophilic interactions with the purine heterocycles. The different nucleic-acid bases furthermore offer a range of steric and electronic factors to exploit. Coordination of a metal complex to the N7 nitrogen atom of a purine, for example, would position other coordinated ligands on the metal center for close hydrogen bonding to the O6 oxygen atom of guanine, but would lead to clashes with the amine hydrogen atoms of adenine.

The monomeric units strung together in a polynucleotide furthermore provide an array of polymeric conformers. Figure 8.2A (See color plate section, pages $C$-14, C-15.) shows three crystallographically characterized structures of double-helical DNA oligonucleotides, ${ }^{2-4}$ Figure $8.2 \mathrm{~B}$ a schematic illustration of other conformations of DNA, and Figure $8.2 \mathrm{C}$ the crystal structure ${ }^{5}$ of yeast tRNA $^{\text {Phe }}$. In double-helical DNA, ${ }^{1}$ the two antiparallel polynucleotide strands 


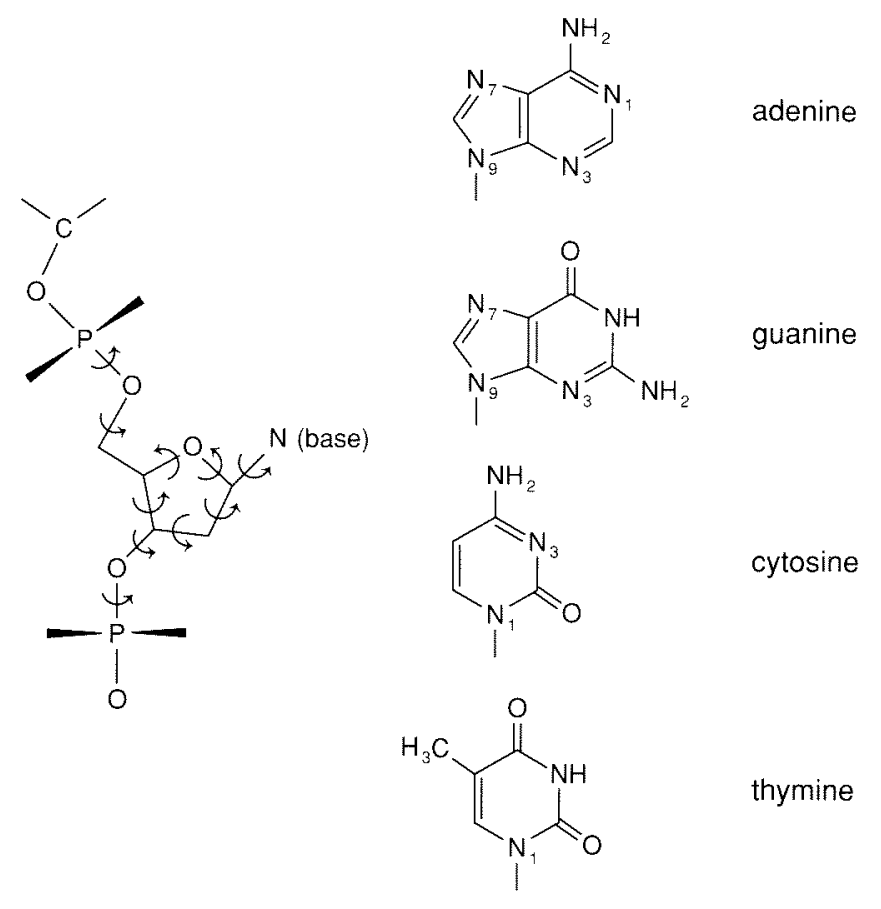

Figure 8.1

Illustration of a mononucleotide unit. Arrows indicate the various torsional angles within each unit that together generate the wide range of conformations available in the polymer. Also shown are the individual bases as well as the commonly employed numbering scheme.

are intertwined in a helix, stabilized through Watson-Crick hydrogen bonding between purines and pyrimidines, and through $\pi-\pi$ stacking interactions among the bases arranged in the helical column. There are electrostatic repulsions between the anionic phosphate backbones of the polymer, causing a stiffening; each double-helical step has two formal negative charges. An atmosphere of metal ions condensed along the sugar-phosphate backbone serves partially to neutralize these electrostatic interactions. In the B-DNA conformation, the bases are stacked essentially perpendicular to the helical axis, and the sugars are puckered in general, with a $\mathrm{C}^{\prime}$-endo geometry (the $\mathrm{C} 2{ }^{\prime}$ carbon is to the same side as the $\mathrm{C}^{\prime}$ position relative to a plane in the sugar ring defined by the $\mathrm{Cl}^{\prime}, \mathrm{C}^{\prime}$, and $\mathrm{O}$ atoms). This conformer yields a right-handed helix with two distinct, well-defined grooves, termed the major and minor. The A-form helix, while still right-handed, is distinctly different in structure. The sugar rings are puckered generally in the $\mathrm{C}^{\prime}$-endo conformation, causing the bases to be pushed out from the center of the helix toward the minor groove, and tilted relative to the helix perpendicular by almost $20^{\circ}$. What results is a shorter and fatter helix than the B-form; the helical pitch is $28.2 \AA$ in A-DNA for an 11-residue helix and $33.8 \AA$ for a 10 -residue helix in B-DNA. The A-form helical shape is best characterized by the very shallow minor groove surface; what was the major groove in the B-form has been pulled deeply into the interior of the A-conformer 
and is really not accessible to binding by small molecules in solution. Transitions to the A-conformation are promoted by hydrophobic solvents or solutions of high ionic strength. The Z-conformation is perhaps most distinctive, owing to its left-handed helicity. ${ }^{4}$ The conformer was dubbed Z-DNA because of the zig-zag in the helix. Alternations both in sugar puckering, between $\mathrm{C} 2{ }^{\prime}$-endo and $\mathrm{C} 3$ '-endo, and in the rotation of the base about the glycosidic bond, anti or syn relative to the sugar, are evident, and lead to a dinucleoside repeating unit versus a mononucleoside repeat in the A- and B-helices. Alternating purinepyrimidine sequences have the highest propensity to undergo transitions into the Z-form. It is actually this syn conformation of purines that leads to the lefthanded helicity of the polymer. But it is not only its left-handedness that distinguishes the Z-conformation. The polymer is long and slender (the pitch is $45 \AA$ for a 12-residue helix), and the major groove is a shallow and wide, almost convex, surface, whereas the minor groove is narrowed into a sharp and small crevice.

These crystal structures, shown in Figure 8.2A (see color plate section, page C-15), in fact each represent a family of conformations. The bases in a base pair often do not lie in the same plane, but are instead propeller-twisted with respect to one another. The local unwinding of the helix and tilting of the base pairs furthermore tend to vary with the local nucleic-acid sequence so as to maximize stacking or hydrogen-bonding interactions among the bases. Hence there is a variety of structures within each conformational family. Our understanding of these structural variations as a function of solution conditions and importantly of local sequence is still quite poor. But surely these structural variations affect and are affected by the binding of metal ions and complexes.

Even less defined structurally are other conformations of DNA, some of which are illustrated schematically in Figure $8.2 \mathrm{~B}$ (see color plate section, page C-15). Double-helical DNA can bend, ${ }^{6}$ form loops and cruciforms, ${ }^{7}$ and fold back on itself into intramolecular triple helices, termed H-DNA. ${ }^{8}$ At the ends of chromosomes, four strands may even come together in a unique conformation. These structures, characterized thus far by means of biochemical techniques, arise because of sequence and local torsional stress, or supercoiling. Many of these structures are stabilized by the binding of highly charged metal ions, probably because the highly charged metal center in a small volume can neutralize the electrostatic repulsions between polyanionic strands that are bundled together. Metal complexes can furthermore be extremely useful in targeting and characterizing these structures, as we will see. In chromosomes the DNA is packaged by histone proteins into even tighter bundles, with helical segments wrapped about the basic proteins to form superhelical nucleosomal units which are then arranged like beads on a string of more loosely packed DNA. ${ }^{9}$

This complexity in DNA structure is in fact small compared to that of RNA. Figure 8.2C (see color plate section, page C-15) shows the first crystallographically characterized structure ${ }^{5}$ of an RNA polymer, yeast tRNA ${ }^{\text {Phe }}$. Ostensibly single-stranded RNAs do not exist as random coils, but instead fold up into well-defined three-dimensional structures, much like proteins. The structural variety, of course, bears some resemblance to that found in DNAs. Double-helical 
regions in the tRNA are A-like in conformation; helices fold together as one might imagine to occur in cruciforms, and even triple-helical segments are evident where three strands fold together in the polymer. But overall our ability to characterize structures of RNA thus far is lower than that with DNAs. RNAs are less stable in solution than is DNA, and fewer chemical as well as enzymatic tools are available for structural characterization. Yet the recent discovery of ribozymes, ${ }^{10}$ the finding that RNAs can indeed catalyze nucleolytic reactions, makes our need to understand these structures even greater. Again transitionmetal chemistry may participate in stabilizing, promoting, and probing these structures.

\section{B. Fundamental Interactions with Nucleic Acids}

Metal ions and complexes associate with DNA and RNA in a variety of ways, as illustrated in Figure 8.3. Both strong covalent interactions and weak noncovalent complexes are observed. ${ }^{11}$ Each may yield a significant perturbation in the nucleic acid and/or may be exploited to obtain a site-specific response. Clearly there are some general guidelines, based on principles of coordination chemistry, that may be helpful in sorting out these interactions.

\section{Coordination}

Most prevalent among covalent complexes with DNA are those involving coordination between soft metal ions and nucleophilic positions on the bases. The structure ${ }^{12}$ of $c i s-\left(\mathrm{NH}_{3}\right)_{2} \mathrm{Pt}-\mathrm{dGpG}$ is an example: its platinum center coordinates to the $\mathrm{N} 7$ position of the guanine bases. In terms of interactions with the full polynucleotide, it is likely that the cis-diammineplatinum center, with two coordination sites available, would yield an intrastrand crosslink between neighboring guanine residues on a strand (see Chapter 9). Other nucleophilic sites targeted by soft metal ions on the bases include the N7 position of adenine, the N3 position on cytosine, and the deprotonated N3 position on thymine and uracil. ${ }^{12,13}$ Some additional covalent binding to the N1 positions of the purines has also been observed. Indeed, coordination by the metal to one site on the heterocyclic base lowers the $\mathrm{p} K_{a}$ and increases the metal-binding affinity to secondary sites. It is noteworthy, however, that in base-paired double-helical DNA only the N7 positions on the purines are easily accessible in the major groove of the helix. Base binding at the purine N7 position is, of course, not limited to soft metal ions such as $\mathrm{Pt}(\mathrm{II}), \mathrm{Pd}(\mathrm{II})$, and $\mathrm{Ru}(\mathrm{II})$. Coordination at these sites has been evident also with first-row transition-metal ions such as $\mathrm{Cu}(\mathrm{II})$ and $\mathrm{Zn}(\mathrm{II}){ }^{13}$ For these, as is consistent with basic coordination chemistry, the lability of complexes formed is higher.

Transition-metal ions with decreasing softness are capable of coordinating also to the phosphate oxygen atoms. The ionic versus covalent character of these complexes clearly depends on the metal ions involved. In a classic study, examining the melting temperature of double-helical DNA in the presence of dif- 


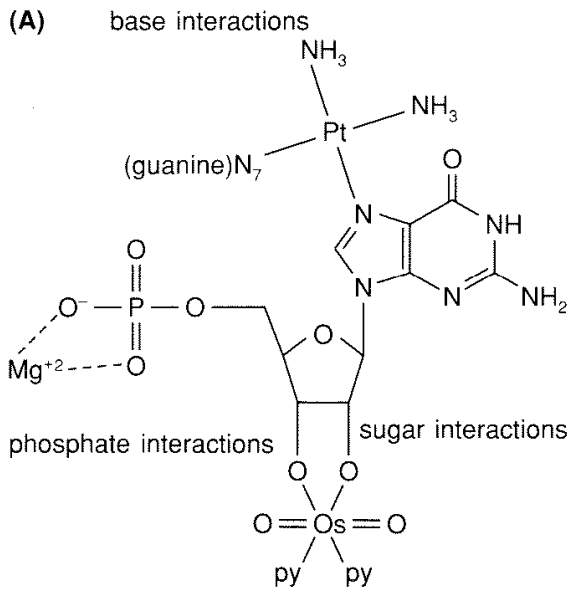

(B)

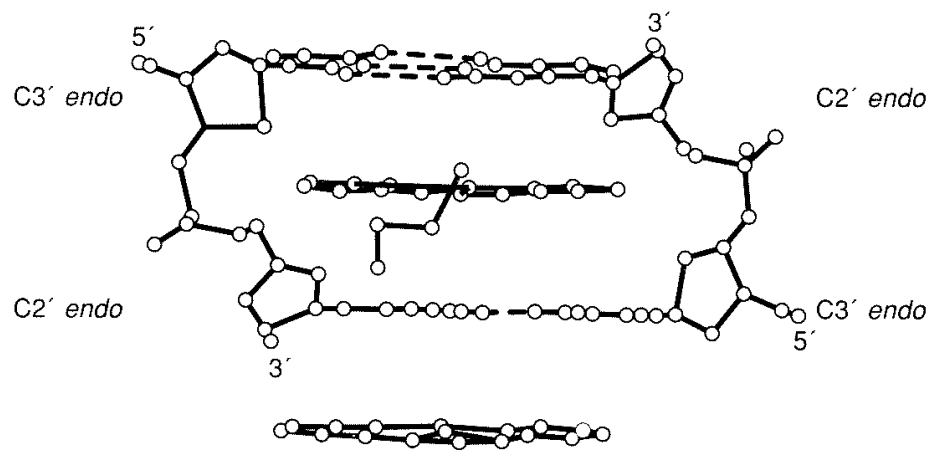

intercalation
(C)

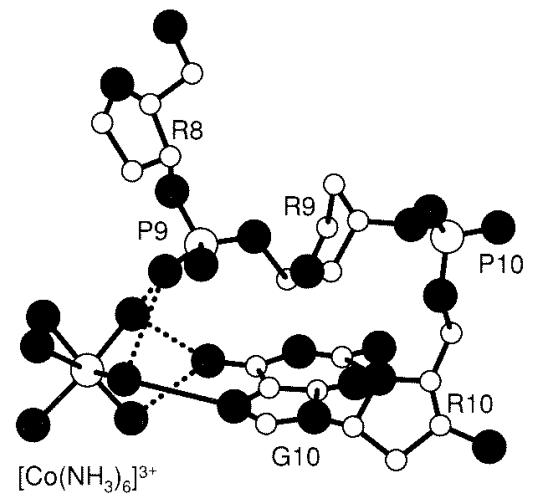

hydrogen bonding

Figure 8.3

Covalent and noncovalent binding modes of metal complexes with DNA. (A) Representative covalent interactions. Shown schematically are examples of coordination to the DNA base, sugar, and phosphate moieties given by the covalent binding of cis-(diammine)platinum to the N7 nitrogen atom of neighboring guanine residues, the formation of an osmate ester with ribose hydroxyl groups, and the primarily electrostatic association between $\mathrm{Mg}\left(\mathrm{H}_{2} \mathrm{O}\right)_{6}{ }^{2+}$ and the guanosine phosphate, respectively. (B) Noncovalent intercalative stacking of a metal complex.

Shown is the crystal structure ${ }^{20 b}$ of (terpyridyl)(2-hydroxyethanethiolate)platinum(II) intercalated and stacked above and below the base-paired dinucleotide $\mathrm{d}(\mathrm{CpG})$. (C) An illustration of hydrogen bonding of coordinated ligands. Shown is a partial view of the crystal structure ${ }^{19}$ of $\mathrm{Z}$-form $\mathrm{d}(\mathrm{CG})_{3}$ with $\mathrm{Co}\left(\mathrm{NH}_{3}\right)_{6}{ }^{3+}$ hydrogen-bonded both to the guanine base $(\mathrm{GlO})$ and phosphate backbone (P9). 


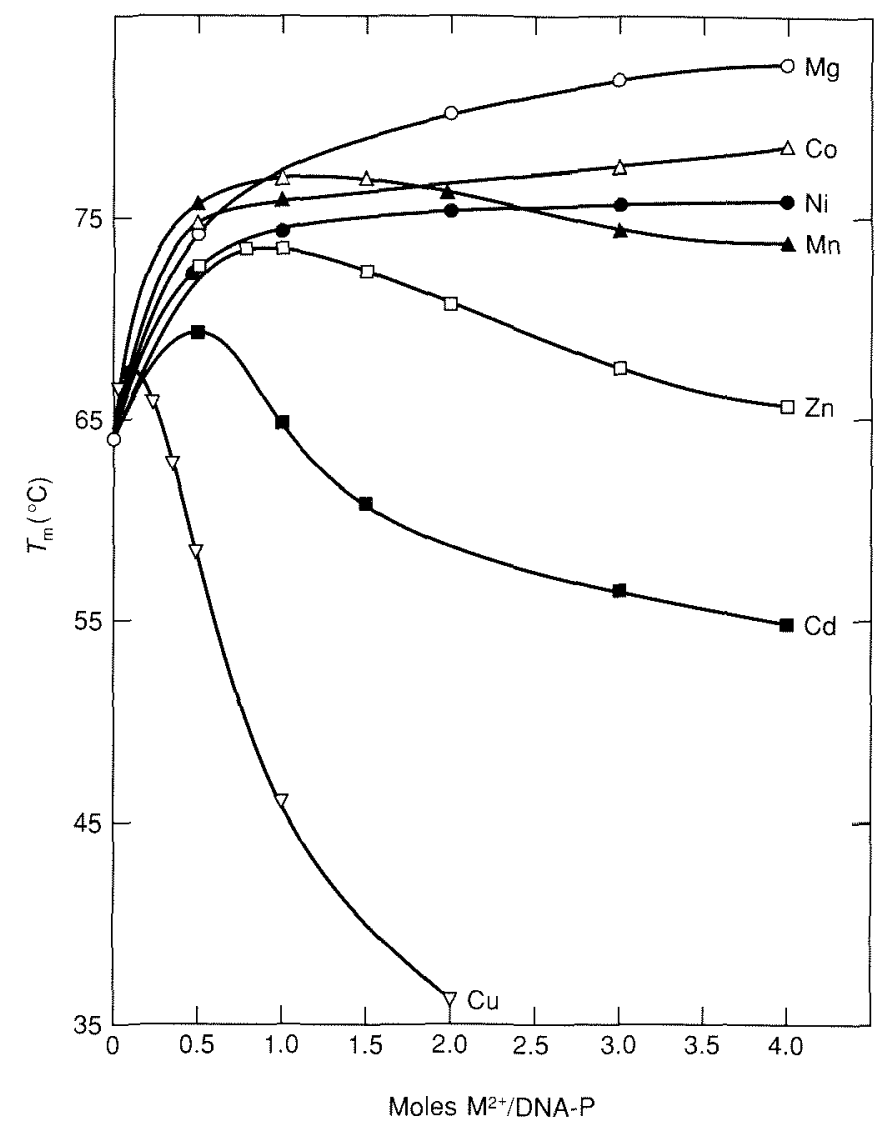

Figure 8.4

The effects of various metal ions on the melting temperature $\left(T_{\mathrm{m}}\right)$ of calf thymus DNA. ${ }^{14}$ Reproduced with permission from Reference 14.

ferent metal ions and as a function of their concentration, Eichhorn and coworkers established the preference of the metal ions for base versus phosphate binding (Figure 8.4). ${ }^{14}$ The preference for phosphate over base association was found to decrease in the order $\mathrm{Mg}(\mathrm{II})>\mathrm{Co}(\mathrm{II})>\mathrm{Ni}(\mathrm{II})>\mathrm{Mn}(\mathrm{II})>\mathrm{Zn}(\mathrm{II})>\mathrm{Cd}(\mathrm{II})>$ $\mathrm{Cu}$ (II). This series arises from examination of DNA helix-melting temperatures, since base interactions in general should destabilize the helical form [except where interstrand crosslinking occurs, as may happen with $\mathrm{Ag}(\mathrm{I})]$, whereas phosphate coordination and neutralization would increase the helix stability and hence the melting temperature.

Also of interest, but less common, are covalent interactions with the sugar moiety. ${ }^{15,16}$ Although the pentose ring in general provides a poor ligand for metal ions, osmate esters can form quite easily across the $\mathrm{C} 2^{\prime}-\mathrm{C}^{\prime}$ positions in ribose rings. This particular interaction has been suggested as a basis for heavymetal staining of RNA. In fact, $\mathrm{OsO}_{4}$ is not restricted in its reactivity with the 
sugar positions. Cisoid osmate esters form as well upon reaction of $\mathrm{OsO}_{4}$ across the electron-rich $\mathrm{C5}$-C6 double bonds of accessible pyrimidines on DNA.

\section{Intercalation and hydrogen bonding}

But important interactions of metal complexes with polynucleotides are not restricted to those involving direct coordination of the metal center to the polymer. Instead, an abundance of highly selective interactions arise from an ensemble of weaker noncovalent interactions between the ligands of coordinatively saturated metal complexes and the nucleic acid. Two primary examples of noncovalent association are given by metallointercalation and hydrogen-bonding interactions of coordinated ligands. ${ }^{17,18}$ Planar aromatic heterocyclic ligands such as phenanthroline and terpyridine can stack in between the DNA base pairs, stabilized through dipole-dipole interactions. Here, depending on the complex and its extent of overlap with the base pairs, the free energy of stabilization can vary from $\sim 2$ to $10 \mathrm{kcal}$. Nonintercalative hydrophobic interactions of coordinated ligands in the DNA grooves also can occur, as we will see. Hydrogenbonding interactions of coordinated ligands with the polynucleotide are quite common, and arise in particular with the phosphate oxygen atoms on the backbone. With cobalt hexaammine, for example, hydrogen bonding to an oligonucleotide occurs between the ammine hydrogens and both phosphate oxygen atoms and purine bases. ${ }^{19}$

A mix of covalent and noncovalent interactions is also possible. With cisdiammineplatinum(II) coordinated to the guanine N7 position, the ammine ligands are well-poised for hydrogen-bonding interactions with the phosphate backbone. ${ }^{12}$ The steric constraints on the molecule must be considered, however. With $\mathrm{Pt}$ (terpy) $\mathrm{Cl}^{+}$, both intercalation of the terpy ligand and direct coordination of the platinum center (after dissociation of the coordinated chloride) are available, but not simultaneously; coordination of the platinum to the base would likely position the terpyridyl ligand away from the base stack in the DNA major groove, precluding intercalation. ${ }^{20}$ Sigel and coworkers ${ }^{21}$ have studied the thermodynamics of noncovalent interactions coupled to direct coordination of simple first-row transition-metal complexes with mononucleotides, and these results illustrate well the interplay of weak noncovalent interactions and direct coordination in generating geometric specificity in complex formation.

\section{Fundamental Reactions with Nucleic Acids}

The reactions of transition-metal complexes with polynucleotides generally fall into two categories: (i) those involving a redox reaction of the metal complex that mediates oxidation of the nucleic acid; and (ii) those involving coordination of the metal center to the sugar-phosphate backbone so as to mediate hydrolysis of the polymer. Both redox and hydrolytic reactions of metal complexes with nucleic acids have been exploited with much success in the development of tools for molecular biology. 


\section{Redox chemistry}

The simplest redox reaction with polynucleotides one might consider as an illustration is the Fenton reaction, which indirectly promotes DNA strand scission through radical reactions on the sugar ring. The reaction with $\mathrm{Fe}(\mathrm{EDTA})^{2-}$ is shown in Figure 8.5A. As do other redox-active divalent metal ions, ferrous ion, in the presence of hydrogen peroxide, generates hydroxyl radicals, and in the presence of a reductant such as mercaptoethanol, the hydroxyl radical production can be made catalytic. Although ferrous ion itself does not appear to interact appreciably with a nucleic acid, especially when chelated in an anionic EDTA complex and repelled by the nucleic-acid polyanion, the hydroxyl radicals, produced in appreciable quantities catalytically, attack different sites on the sugar ring, indirectly yielding scission of the sugar-phosphate backbone. One such reaction that has been characterized in some detail is that involving

(A) $\begin{aligned} & \mathrm{Fe}(\text { II })+\mathrm{H}_{2} \mathrm{O}_{2} \longrightarrow \mathrm{Fe}(\text { III })+\mathrm{OH} \cdot+\mathrm{OH}^{-} \\ & \mathrm{Fe}(\mathrm{III})+\mathrm{e}^{-} \longrightarrow \mathrm{Fe}(\mathrm{II})\end{aligned}$

(B)

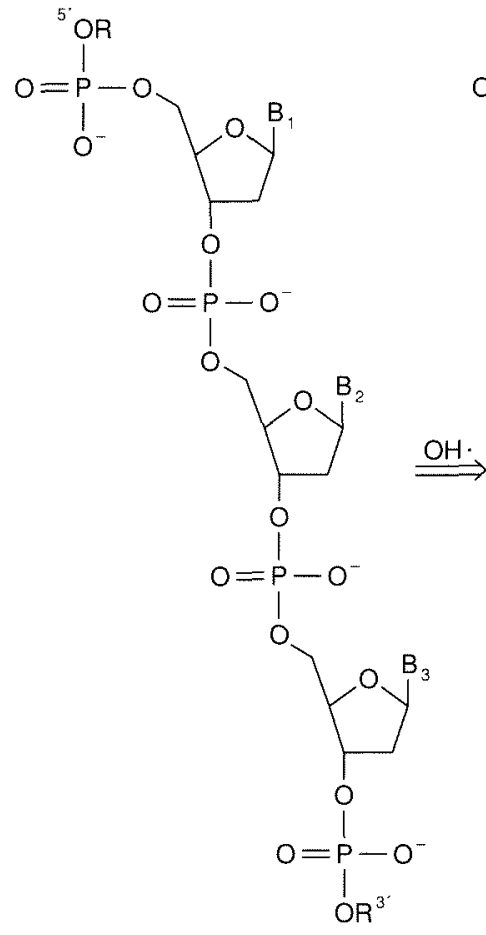<smiles>O=P([O-])([O-])OCC1OC(Br)CC1OP(=O)([O-])[O-]</smiles><smiles>CCCCCCCCCCCOP(=O)([O-])OCC1OC2[B]CC21</smiles>
nucleic-acid base $\left(B_{2}\right)$<smiles>O=P([O-])(O)OCC1OC2CC1C2OP(=O)([O-])O</smiles>

\section{Figure 8.5}

An illustration of DNA strand cleavage mediated by hydroxyl radicals produced by the Fenton reaction (A) of Fe(EDTA) ${ }^{2-}$ with hydrogen peroxide. The cleavage scheme (B) shows the products obtained as a result of initial $\mathrm{C}^{\prime}-\mathrm{H}$ abstraction by the hydroxyl radicals. 
hydroxyl radical reaction at the $\mathrm{C}^{\prime}$ position, the position most accessible to the diffusible radical in the minor groove of the helix. ${ }^{22}$ As illustrated in Figure $8.5 \mathrm{~B}$, the products of this reaction include a $5^{\prime}$-phosphate, a mixture of $3^{\prime}$ phosphate and phosphoglycolates, and a mixture of free bases and base propenals. Reactions of the hydroxyl radical at other sites on the sugar ring are now being identified as well by isotope-labeling studies. Comparable reactions with RNA have also been described. ${ }^{23}$

The application of this Fenton chemistry to promote site-specific or sequence-neutral cleavage of DNA was first demonstrated ${ }^{24}$ by Dervan and coworkers, and has provided the basis for the design of a tremendous range of new and valuable DNA cleavage agents. The development of this chemistry was originally based on modeling Fe-bleomycin, a natural product with antitumor and antibiotic activity, which binds and cleaves DNA. ${ }^{25}$ The chemistry mediated by Fe-bleomycin, as we will discuss later, is likely to be far more complex, however, involving direct reaction of an intimately bound ferryl intermediate species with the nucleic acid, rather than net oxidation of the sugar mediated by a diffusing hydroxyl radical. Other metal ions such as $\mathrm{Cu}$ (II) can also promote redox-mediated cleavage of $\mathrm{DNA}^{26,27}$ through reactions on the sugar ring; whether the oxidizing radical is still coordinated to the metal or is a dissociated and diffusing species is a topic of much debate. ${ }^{26}$

Metal ions can also be used to generate other oxidizing intermediates in aerated aqueous solution, such as superoxide ion and singlet oxygen. DNA strandcleavage reactions mediated by superoxide have not thus far been demonstrated, however. Singlet oxygen may be produced by photosensitization of $\mathrm{Ru}(\mathrm{phen})_{3}{ }^{2+}$, and indeed photolysis of $\mathrm{Ru}(\mathrm{phen})_{3}{ }^{2+}$ bound to DNA yields oxygen-dependent, alkaline-sensitive strand cleavage. ${ }^{28,29}$ For singlet oxygen, the oxidation occurs on the nucleic-acid base rather than on the sugar ring. As such, the reaction varies with base composition; guanine residues are most reactive. Furthermore, since the primary lesion is that of a base modification, piperidine treatment, or other weakly basic conditions, are needed to convert the base lesion into a strandscission event.

Another scheme for oxidative cleavage of DNA mediated by metal complexes involves formation of a coordinated ligand radical bound to the helix that directly abstracts a hydrogen atom from the sugar ring. The photoreaction of $\mathrm{Rh}$ (phen) ${ }_{2} \mathrm{phi}^{3+}$ (phi $=9,10$-phenanthrenequinone diimine) exemplifies this strategy. ${ }^{30}$ Here photolysis promotes a ligand-to-metal charge transfer with formation of a phi-centered radical. Isotope-labeling studies and product analysis have shown that this phi radical bound intercalatively in the major groove of DNA directly abstracts the $\mathrm{C}^{\prime} \cdot \mathrm{H}$ (which sits in the major groove of the helix) $;{ }^{31}$ subsequent hydroxylation or dioxygen addition at this position promotes DNA strand scission without base treatment.

Some potent photooxidants can also produce outer-sphere electron transfer from the DNA. Here it is the guanine bases, likely those stacked with neighboring purines, that are most easily oxidized and hence most susceptible to attack. Again, this base modification requires alkaline treatment to convert the 
lesion to a strand breakage. ${ }^{11 \mathrm{~b}, 17}$ The DNA double helix can furthermore also mediate electron-transfer reactions between bound metal complexes. The DNA polymer has, for example, been shown to catalyze photoinduced electron-transfer reactions between $\mathrm{Ru}(\text { phen })_{3}{ }^{2+}$ and $\mathrm{Co}(\text { phen })_{3}{ }^{3+}$ bound along the DNA strand. ${ }^{32}$ Table 8.1 summarizes different redox reactions of metal complexes bound to DNA.

\section{Hydrolytic chemistry}

Hydrolysis reactions of nucleic acids mediated by metal ions are important elements in natural enzymatic reactions; chemists would like to exploit them in the design of artificial restriction endonucleases. ${ }^{33}$ Hydrolysis reactions of the phosphodiester linkage of polynucleotides appear preferable to redox-mediated cleavage reactions, since in the hydrolytic reaction all information is preserved. In redox cleavage by sugar oxidation, for example, both a sugar fragment and free nucleic-acid base are released from the polymer, and, in contrast to hydrolytic chemistry, the direct religation of the fragments becomes practically impossible.

Table 8.1

Examples of metal complexes that cleave DNA through redox chemistry.

\begin{tabular}{|c|c|c|c|c|c|}
\hline Complex & Target $^{\mathrm{a}}$ & Chemistry $^{\mathrm{b}}$ & Diffusibility $^{\mathrm{c}}$ & DNA Binding ${ }^{d}$ & Site Selectivity \\
\hline $\mathrm{Fe}(\mathrm{EDTA})^{2-}$ & sugar & $\mathrm{OH} \cdot$, Fenton & diffusible & none & none \\
\hline MPE-Fe(II) & $\begin{array}{l}\text { sugar } \\
\left(\mathrm{C}^{\prime}-\mathrm{H}\right)\end{array}$ & $\mathrm{OH} \cdot$, Fenton & diffusible & sequence-neutral & none \\
\hline $\mathrm{Co}\left(\mathrm{NH}_{3}\right)_{6}^{3+*}$ & base & $\begin{array}{l}\text { photoelectron } \\
\text { transfer }\end{array}$ & $\mathrm{f}$ & hydrogen-bonding & $5^{\prime}-\mathrm{G}-$ pur-3' \\
\hline $\mathrm{Cu}(\text { phen })_{2}{ }^{+}$ & sugar & $\mathrm{Cu}^{2+}-\mathrm{OH}$ & slight & AT-rich & AT-rich \\
\hline Mn-Porphyrin & sugar & $\mathrm{M}=\mathrm{O}$ & none & AT-rich & AT-rich \\
\hline $\mathrm{U}\left(\mathrm{O}_{2}\right)\left(\mathrm{NO}_{3}\right)_{2} *$ & f & f & diffusible & f & none \\
\hline $\operatorname{Ru}(\mathrm{TMP})_{3}{ }^{2+*}$ & base & ${ }^{1} \mathrm{O}_{2}$ & diffusible & A-form & A-form, $\mathrm{G}$ \\
\hline $\operatorname{Ru}(\text { phen })_{3}{ }^{2+*}$ & base & ${ }^{1} \mathrm{O}_{2}$ & diffusible & sequence-neutral & $\mathrm{G}$ \\
\hline $\mathrm{Co}(\mathrm{DIP})_{3}{ }^{3+*}$ & sugar & ligand radical & none & Z-form (non-B) & $Z$-form (non-B) \\
\hline $\operatorname{Rh}(\mathrm{DIP})_{3}{ }^{3+*}$ & sugar & ligand radical & none & $\mathrm{Z}$, cruciforms & $\mathrm{Z}$, cruciforms \\
\hline $\operatorname{Rh}(\text { phen })_{2}$ phi $^{3+*}$ & $\begin{array}{l}\text { sugar } \\
\left(\mathrm{C}^{\prime}-\mathrm{H}\right)\end{array}$ & ligand radical & none & open major groove & $5^{\prime}$-pyr-pyr-pur- $3^{\prime}$ \\
\hline $\operatorname{Rh}(\text { phi })_{2} \mathrm{bpy}^{3+*}$ & $\begin{array}{l}\text { sugar } \\
\left(\mathrm{C}^{\prime}-\mathrm{H}\right)\end{array}$ & ligand radical & none & sequence-neutral & none \\
\hline
\end{tabular}

\footnotetext{
${ }^{a}$ DNA may be modified by attack either at the sugar or at the nucleotide base position.

$b$ The reactive species involved in DNA cleavage, if known.

${ }^{c}$ Some reactive species are diffusible, producing broad patterns of DNA damage along the strand. Others are nondiffusible, resulting in cuts at single discrete sites.

d The site of metal complex binding to DNA, if known.

e The sites cleaved by the metal complex.

${ }^{\mathrm{f}}$ Not known.

* Indicates an excited-state reaction requiring photoactivation.
} 
Metal ions can be effective in promoting hydrolysis of the phosphodiester, since they can function as Lewis acids, polarizing the phosphorus-oxygen bond to facilitate bond breakage, and can also deliver the coordinated nucleophile to form the pentacoordinate phosphate intermediate. Figure 8.6 illustrates one crystallographically characterized model system developed by Sargeson and coworkers, where hydrolysis of a model phosphodiester was enhanced dramatically by taking advantage of both the acidic and the nucleophilic characteristics of the bound cobalt(III) species. ${ }^{34} \mathrm{~A}$ whole series of model systems utilizing both cobalt and zinc ions has been designed to explore the hydrolytic reactions of simple phosphodiesters. ${ }^{35}$ This strategy coupled to a DNA binding functionality has also been exploited, albeit inefficiently, in the hydrolytic cleavage of doublehelical DNA by $\mathrm{Ru}(\mathrm{DIP})_{2} \mathrm{Macro}$ with $\mathrm{Zn}^{2+}, \mathrm{Cd}^{2+}$, or $\mathrm{Pb}^{2+}$ added in situ ${ }^{36} \mathrm{In}$ this complex (see Figure 8.6), the central portion of the molecule, held together by the ruthenium(II), is responsible for DNA binding. Tethered onto the coordinatively saturated ruthenium complex are two diethylenetriamine functionalities (in the Macro ligand), however, and these serve to coordinate hydrolytically active metal ions such as $\mathrm{Zn}(\mathrm{II})$ and Co(II), which promote DNA hydrolysis once delivered to the sugar-phosphate backbone by the DNA-binding domain.

Perhaps simpler and certainly better understood are the hydrolytic reactions of RNAs mediated by metal ions. More than twenty years ago Eichhorn and coworkers showed that simple metal ions such as $\mathrm{Zn}$ (II) and $\mathrm{Pb}$ (II) promote the hydrolysis of RNA. ${ }^{37}$ Figure 8.6 illustrates also the crystallographically characterized site-specific hydrolyis in tRNA by plumbous ion. ${ }^{38}$ In tRNA, Pb(II) occupies three quite specific high-affinity binding sites, and at one of these sites, the metal ion becomes poised to promote strand cleavage. The crystal structure with bound $\mathrm{Pb}^{2+}$ suggests that the lead-coordinated hydroxide ion deprotonates the $2^{\prime}$-hydroxyl of one residue, so that the resulting $2^{\prime}$-oxygen nucleophile may attack the phosphate to give a pentavalent intermediate that decays to form the $2^{\prime}, 3^{\prime}$-cyclic phosphate and, after reprotonation, the $5^{\prime}$-hydroxide. This very specific cleavage reaction is already being used by biologists as a tool in probing structures of mutant tRNAs, since the reaction is exquisitely sensitive to the stereochemical alignment of the nucleic-acid residues, phosphate backbone, and associated metal ion. In hydrolytic reactions on RNA, it is commonly considered, though certainly not established, that the job of the metal ion may be simpler than with DNA, since the ribose provides a nearby nucleophile already in the $2^{\prime}$-hydroxide. The reaction of tRNA with $\mathrm{Pb}$ (II) nonetheless illustrates how a metal ion may be utilized in promoting highly specific chemistry on a nucleic-acid polymer.

Last, it must be mentioned that metal coordination to the purine $\mathrm{N} 7$ position can also indirectly promote strand cleavage, although not through direct hydrolytic reaction on the sugar-phosphate backbone. Metal ions such as $\mathrm{Pd}^{2+}$ and $\mathrm{Cu}^{2+}$, through coordination at $\mathrm{N} 7$, promote depurination. The depurinated site then becomes easily susceptible to hydrolysis upon treatment with mild base. 
(A)<smiles>CCCC1OP(=O)(P)OP(=O)(O)OC1C</smiles>

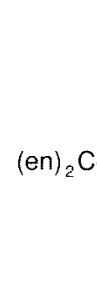

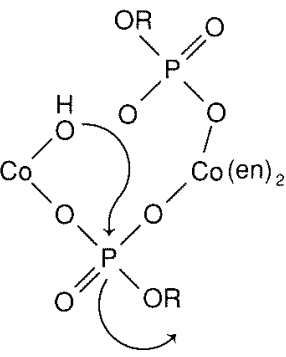

en = ethylene diamine

(B)

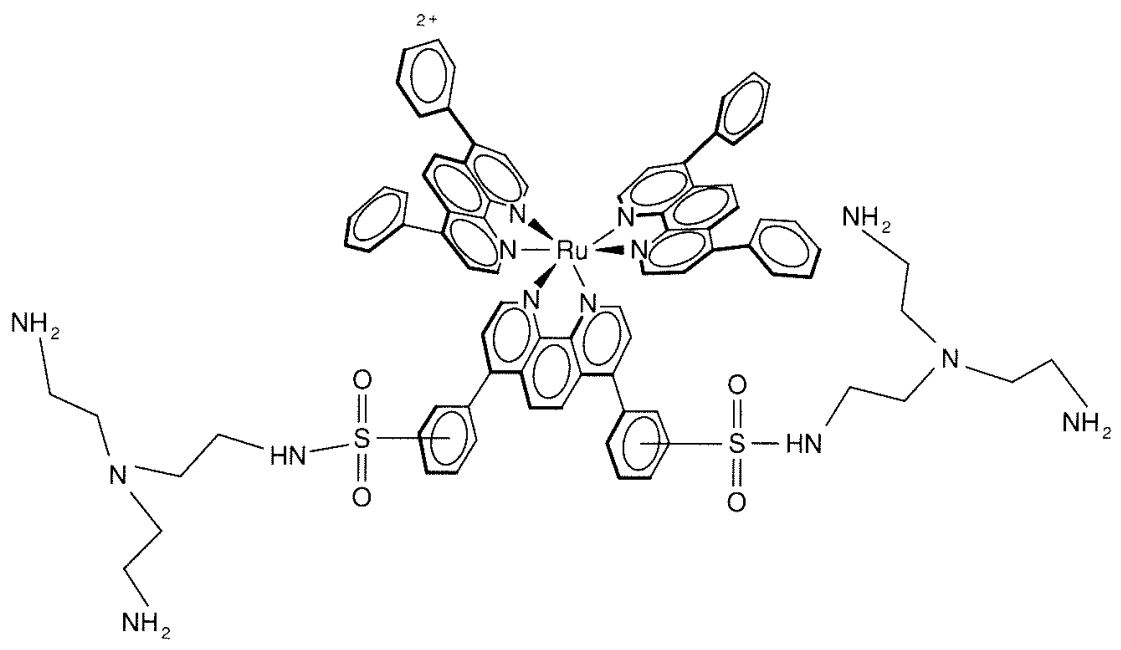

(C)

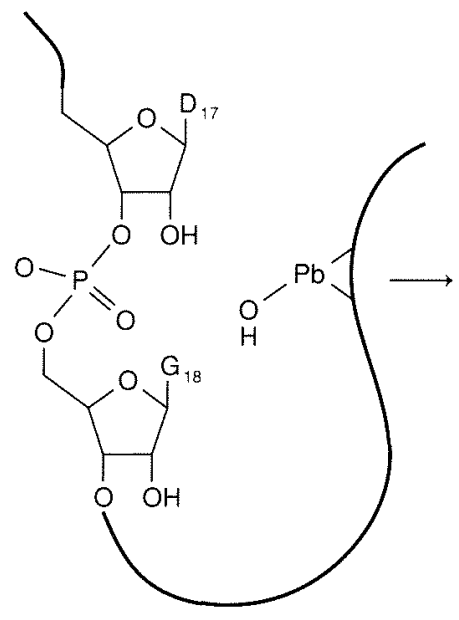<smiles></smiles>

$\mathrm{Pb}\left(\mathrm{H}_{2} \mathrm{O}\right)_{5}^{2+}$

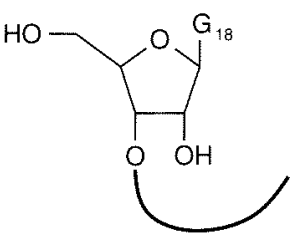

Figure 8.6

Hydrolysis reactions catalyzed by metal ions and complexes. (A) Illustration of a phosphate ester hydrolysis in a binuclear model complex catalyzed by coordinated cobaltic ions, with one metal ion functioning as a Lewis acid and the other functioning to deliver the coordinated hydroxide. $^{34}$ (B) Ru(DIP) ${ }_{2}$ Macro, a metal complex constructed to contain a central DNA-binding domain $\left(\mathrm{Ru}(\mathrm{DIP})_{3}{ }^{2+}\right.$ ) with two tethered amine arms to chelate additional metal ions $\left(\mathrm{Zn}^{2+}\right)$ to deliver to the sugar-phosphate backbone and promote hydrolytic strand cleavage. ${ }^{36}$ (C) RNA site-specificially hydrolyzed by lead ion. Diagram of the proposed mechanism of sugar-phosphate backbone cleavage between residue $D_{17}$ and $G_{18}$ in yeast $R A^{\text {Phe }}{ }^{38}$ 


\section{A CASE STUDY: TRIS(PHENANTHROLINE) METAL COMPLEXES}

Now we may examine in detail the interaction of one class of metal complexes with nucleic acids, how these complexes bind to polynucleotides, the techniques used to explore these binding interactions, and various applications of the complexes to probe biological structure and function. Tris(phenanthroline) metal complexes represent quite simple, well-defined examples of coordination complexes that associate with nucleic acids. Their examination should offer a useful illustration of the range of binding modes, reactivity, techniques for study, and applications that are currently being exploited and explored. In addition, we may contrast these interactions with those of other transition-metal complexes, both derivatives of the tris(phenanthroline) family and also some complexes that differ substantially in structure or reactivity.

\section{A. Binding Interactions with DNA}

Tris(phenanthroline) complexes of ruthenium(II), cobalt(III), and rhodium(III) are octahedral, substitutionally inert complexes, and as a result of this coordinative saturation the complexes bind to double-helical DNA through a mixture of noncovalent interactions. Tris(phenanthroline) metal complexes bind to the double helix both by intercalation in the major groove and through hydrophobic association in the minor groove. ${ }^{11 \mathrm{~b}, 40}$ Intercalation and minor groove-binding are, in fact, the two most common modes of noncovalent association of small molecules with nucleic acids. In addition, as with other small molecules, a nonspecific electrostatic interaction between the cationic complexes and the DNA polyanion serves to stabilize association. Overall binding of the tris(phenanthroline) complexes to DNA is moderate $(\log K=4) .{ }^{41}$

The extent of intercalative versus groove binding is seen to depend upon environmental conditions, such as temperature and ionic strength, the charge of the metal center, and the DNA base sequence; groove binding is favored at AT-rich sequences. ${ }^{41}$ Second-generation mixed-ligand derivatives of the tris(phenanthroline) series have been prepared, and their interactions with DNA have provided useful insight into the factors important for promoting either intercalation or groove binding. ${ }^{42}$ Aromatic heterocyclic ligands with increased surface areas that are planar bind DNA with increasing avidity through intercalation, irrespective of the charge on the metal center. Intercalative binding constants greater than $10^{7} \mathrm{M}^{-1}$ can be easily achieved with planar heterocyclic ligands that jut out from the metal center. Not surprisingly, complexes containing ligands of increasing hydrophobicity that are not planar favor minor-groove binding. ${ }^{28}$

Critically important as well in determining the binding mode is the chirality of the metal complex ${ }^{40}$ Intercalation into the right-handed helix favors the $\Delta$ isomer, whereas groove binding favors the $\Lambda$-isomer. Figure 8.7 illustrates these symmetry-selective interactions. In intercalation, we consider that one phenan- 


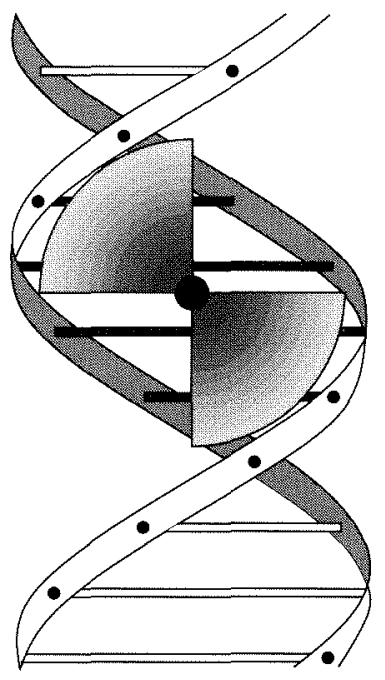

$\Lambda$

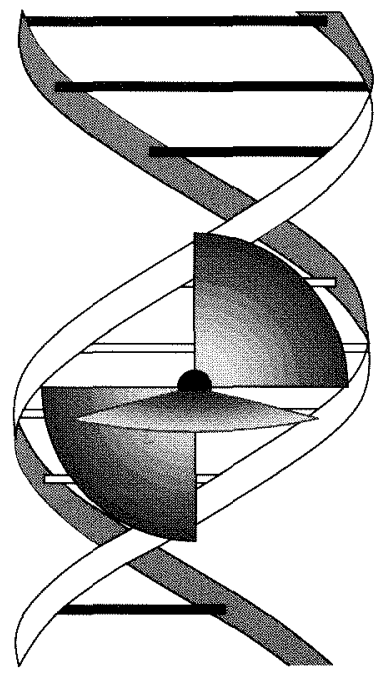

$\Lambda$

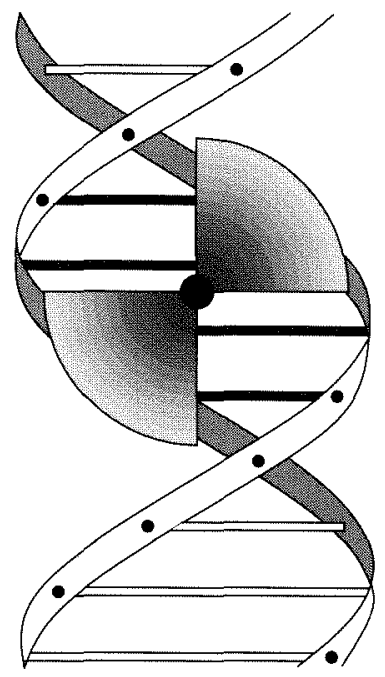

$\Delta$

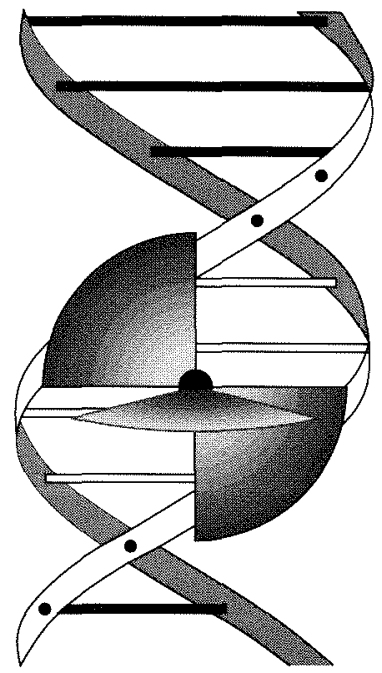

$\Delta$

Figure 8.7

Enantiomeric discrimination in binding to DNA. Shown above is the basis for the preference for $\Delta$-Ru(phen $)_{3}{ }^{2+}$ upon intercalation and $\Lambda$-Ru(phen $)_{3}{ }^{2+}$ for surface binding against a right-handed helix. With intercalation (top) the symmetry of the metal complex matches the symmetry of the helix; steric interactions preclude a close association of the $\Lambda$-isomer. With groove binding (bottom), where the metal complex binds against the minor-groove helical surface, complementary symmetries are required, and it is the $\Lambda$-isomer that is preferred. 
throline inserts and stacks in between the base pairs, essentially perpendicular to the helix axis. For the $\Delta$-isomer, once intercalated, the ancillary non-intercalated ligands are aligned along the right-handed groove of the helix. For the $\Lambda$-isomer, in contrast, with one ligand intercalated, the ancillary ligands are aligned in opposition to the right-handed groove, and steric interactions become evident between the phenanthroline hydrogen atoms and the phosphate oxygen atoms. Increasing the steric bulk on these phenanthrolines furthermore increases the enantioselective preference for intercalation of the $\Delta$-isomer ${ }^{40,43}$ For intercalation, then, the chiral discrimination depends on matching the symmetry of the metal complex to that of the DNA helix. For groove binding, where the metal complex is thought to bind against the helix, instead it is a complementary symmetry that is required. In our model for groove binding of the tris(phenanthroline) metal complex, two phenanthroline ligands are likely bound against the right-handed helical groove, stabilized through hydrophobic association. For the $\Lambda$-isomer, bound in this fashion, the ligands lie against and complement the right-handed groove; with the $\Delta$-isomer, the ligands oppose the groove, and no close surface contacts are made.

Intercalation of metal complexes in DNA is not uncommon. Lippard and coworkers first established metallointercalation by $\mathrm{Pt}(\mathrm{II})$ complexes in the 1970s. ${ }^{18,20}$ Square-planar platinum(II) complexes containing the terpyridyl ligand were shown to intercalate into DNA. In an elegant series of $x$-ray diffraction experiments on DNA fibers, Lippard illustrated the requirement for planarity in the complex. ${ }^{18,44}$ Although (phen) $\mathrm{Pt}(\mathrm{en})^{2+}$ and (bpy)Pt(en) ${ }^{2+}$ were shown to intercalate into the helix, $(\mathrm{pyr})_{2} \mathrm{Pt}(\mathrm{en})^{2+}$, with pyridine ligands rotated out of the coordination plane, could not. Complex planarity is in itself insufficient to promote intercalation, however. Cis- $\left(\mathrm{NH}_{3}\right)_{2} \mathrm{PtCl}_{2}$ or even cis- $\left(\mathrm{NH}_{3}\right)_{2} \mathrm{Pt}(\mathrm{en})^{2+}$ does not appear to intercalate into a helix, despite full planarity. Instead, aromatic heterocyclic ligands must be included in order to promote dipole-dipole interactions with the heterocyclic bases stacked in the helix. Indeed, planarity of the full complex is not required. Intercalation is not restricted to coordination complexes that are square planar. The tris(phenanthroline) complexes represented the first examples of "three-dimensional intercalators" and illustrated that octahedral metal complexes could also intercalate into the helix. ${ }^{40,45,46}$ Here one can consider the partial intercalation of one ligand into the helix, providing the remaining ligands on the complex an opportunity to enhance specificity or reactivity at a given site.

Curiously, one unique and apparently general characteristic of metallointercalators is their preference for intercalation from the major groove of the helix. Most small molecules associate with DNA from the minor groove, but metallointercalators, both those that are square planar, such as (terpyridyl)platinum(II) complexes, and those that are octahedral, such as the tris(phenanthroline) metal complexes, appear to intercalate into the major groove. This then mimics quite well the association of much larger DNA-binding proteins with the helix; DNA regulatory proteins generally appear to target the major groove. The reason why metallointercalators favor major groove association is still unclear. 


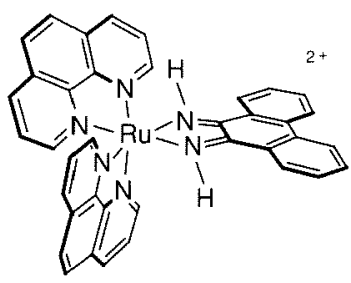

$\left[\mathrm{Ru}(\text { phen })_{2}\right.$ phi $^{2+}$

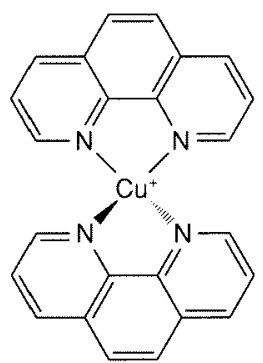

$\mathrm{Cu}(\text { phen })_{2}^{+}$

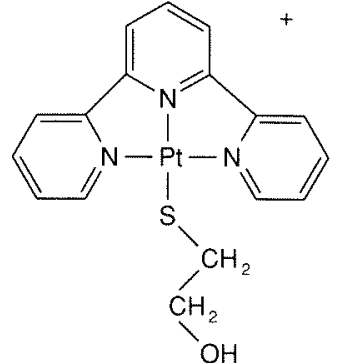

$[(\text { terpy }) \mathrm{Pt}(\mathrm{HEt})]^{+}$

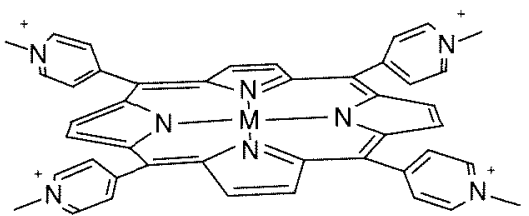

M-T4MPyP

Figure 8.8

Some metal complexes that bind DNA noncovalently primarily through intercalation (top) or binding in the minor groove (bottom). Some metalloporphyrins also primarily associate via intercalation.

Transition-metal complexes with aromatic ligands also generally associate by minor-groove binding or through the mix of intercalative and groove-bound interactions. $\mathrm{Cu}(\text { phen })_{2}{ }^{+}$, a tetrahedral complex, appears to favor minor-groove binding over intercalation. ${ }^{26}$ Perhaps the tetrahedral coordination does not permit appreciable overlap of the phenanthroline ring with the bases in an intercalative mode. Metalloporphyrins, despite their large expanse and the presence commonly of nonplanar substituents, appear to bind to double-helical DNA both by intercalation and by minor-groove binding at AT-rich sequences. ${ }^{47}$ Occupation of the porphyrins by transition-metal ions, such as $\mathrm{Cu}$ (II), which bind axial ligands, leads to the favoring of groove binding over intercalation. Figure 8.8 illustrates some of the complexes that bind DNA noncovalently.

The tris(phenanthroline) metal complexes themselves do not offer an illustration of hydrogen-bonding interactions with the helix, since these ligands lack hydrogen-bonding donors and acceptors, but as mentioned already, hydrogen bonding of coordinated ligands to the helix can add some measure of stabilization, comparable to, but likely no greater in magnitude than, that provided by intercalative stacking, hydrophobic, or dispersive interactions. Indeed, mixed- 
ligand derivatives of the phenanthroline complexes have been prepared that include hydrogen-bonding groups (amides, hydroxyls, and nitro substituents) on the ancillary phenanthroline ligands, and these have shown no greater avidity for double-helical DNA than their counterparts with hydrophobic substituents. ${ }^{42}$ A large number of weak hydrogen-bonding interactions to DNA by one complex can be stabilizing, however, as with, for example, hexaamminecobalt(III) or hexaaquoterbium(III).

Tris(phenanthroline) metal complexes also do not offer an opportunity to explore covalent binding interactions with the helix in greater detail, but these interactions are, in fact, a major focus of Chapter 9, concerned with the mode of action of cisplatin. One derivative of the tris(phenanthroline) series, $\mathrm{Ru}$ (phen) ${ }_{2} \mathrm{Cl}_{2}$, has been shown to bind to DNA covalently. ${ }^{48}$ In aqueous solution the dichlororuthenium(II) complex undergoes hydrolysis to form an equilibrium mixture of bis(phenanthroline) diaquo and chloroaquo species. These species bind covalently to DNA, with preferential reactivity at guanine sites. It is interesting that the same structural deformations in the DNA evident upon binding cis-diammineplatinum units become apparent upon coordination of bis(phenanthroline)ruthenium(II). It is also noteworthy that the chiral preference in coordination is for the $\Lambda$-isomer. As with groove binding, direct coordination to base positions requires a complementary symmetry, with the the $\Lambda$-isomer binding against the right-handed groove. This preference for the $\Lambda$-isomer reaffirms that, rather than noncovalent intercalation (which would favor the $\Delta$ isomer), covalent binding dominates the interaction. The energetic stabilization in direct coordination of the ruthenium(II) center is certainly more substantial than the weaker stabilization derived from intercalation. $\mathrm{Rh}(\mathrm{phen})_{2} \mathrm{Cl}_{2}{ }^{+}$and its derivatives have also been shown to bind covalently to DNA but only upon photoactivation, since light is needed to promote dissociation of the coordinated chloride and substitution of the nucleic acid base as a ligand. ${ }^{49}$

\section{B. Techniques to Monitor Binding}

Many of the same techniques employed in studying the basic chemistry of coordination complexes can be be used in following the binding of transition-metal complexes to nucleic acids, but biochemical methods, with their often exquisite sensitivity, become valuable aids as well in delineating specific binding interactions. Tris(phenanthroline) metal complexes are particularly useful to illustrate this point, since here the metal center in the complex is selected in terms of the technique used for examination.

Coordination complexes are often visibly colored, and these colorations provide a useful and sensitive spectroscopic handle in following fundamental reactions. This notion holds as well with tris(phenanthroline) metal complexes in their interactions with nucleic acids. $\mathrm{Ru}(\mathrm{phen})_{3}{ }^{2+}$ and its derivatives are highly colored because of an intense metal-to-ligand charge-transfer band $\left(\lambda_{\max }=447 \mathrm{~nm}\right.$, $\left.\epsilon=1.9 \times 10^{4} \mathrm{M}^{-1} \mathrm{~cm}^{-1}\right)$. Furthermore, the complexes are highly photoluminescent $\left(\lambda_{\mathrm{em}}=610 \mathrm{~nm}, \tau=0.6 \mu \mathrm{s}\right.$ in aerated aqueous solution). On binding 
to nucleic acids these transitions are perturbed. Hypochromism is observed in the charge-transfer band, and intercalation leads to an increase in lifetime of the charge-transfer excited state. ${ }^{43,46}$ Indeed, single-photon counting experiments show a biexponential decay in emission from $\mathrm{Ru}(\mathrm{phen})_{3}{ }^{2+}$ bound to double-helical DNA. The longer-lived component $(\tau=2 \mu \mathrm{s})$ has been assigned as the intercalated component and the shorter-lived $0.6 \mu$ s component has been attributed to a mixture of free and groove-bound species. These spectroscopic perturbations permit one to define equilibrium-binding affinities for the different components of the interaction as a function of metal-center chirality and under different solution conditions. ${ }^{41}$ One can also follow the polarization of emitted light from the complexes after excitation with polarized light, and these studies have been helpful in describing the dynamics of association of the complexes on the helix. ${ }^{41,43}$ Mixed-ligand complexes of ruthenium(II) show similar spectroscopic perturbations, and these have been used to characterize binding affinities and chiral preferences, as well as the extent of intercalation versus groove binding as a function of ligand substitution on the metal center. ${ }^{42}$ The spectroscopic handle of the metal center therefore affords a range of experiments to monitor and characterize the binding of the metal complexes to polynucleotides.

Binding interactions of metal complexes with oligonucleotides can also be followed by NMR, and here as well the metal center offers some useful characteristics to exploit. As with organic DNA-binding molecules, shifts in the ${ }^{1} \mathrm{H}$ NMR resonances of both the DNA-binding molecule and the oligonucleotide become apparent as a function of increased association with the helix. These shift variations can be used empirically to watch the dynamics of association and to gain some structural insights into the binding modes of the complexes on the helix. These kinds of experiments have been performed with tris(phenanthroline) complexes of ruthenium(II) and rhodium(III), where it was observed that the double-helical oligonucleotide is an exceedingly good chiralshift reagent to separate resonances in an enantiomeric mixture of the tris(phenanthroline) complexes. ${ }^{50}$ For covalent binding molecules, such as cisdiammineplatinum(II), furthermore, the lowering of the $\mathrm{p} K_{a}$ of purine positions and therefore shifting of resonances as a function of coordination to an alternate site has been helpful as well in assigning the sites of covalent binding on the oligonucleotide. ${ }^{51}$ But also in NMR experiments, special advantage can be taken of the metal center. For tris(phenanthroline) metal complexes, ${ }^{1} \mathrm{H}-\mathrm{NMR}$ experiments ${ }^{52}$ were performed on the paramagnetic analogues, $\mathrm{Ni}(\mathrm{phen})_{3}{ }^{2+}$ and $\mathrm{Cr}$ (phen $)_{3}{ }^{3+}$. It was reasonable to assume the binding characteristics would be identical with their respective diamagnetic analogues, $\mathrm{Ru}(\mathrm{II})$ and $\mathrm{Rh}(\mathrm{III})$; yet paramagnetic broadening by the metal complexes of nearby resonances on the oligonucleotide would allow one to deduce where along the helix the complexes associate. Using this method the groove-binding interaction of the complexes was identified as occurring in the minor groove of the helix. Figure 8.9 illustrates the monitoring of DNA binding by tris(phenanthroline) metal complexes using both the luminescence characteristics of ruthenium(II) complexes and the paramagnetic characteristics of nickel(II). 


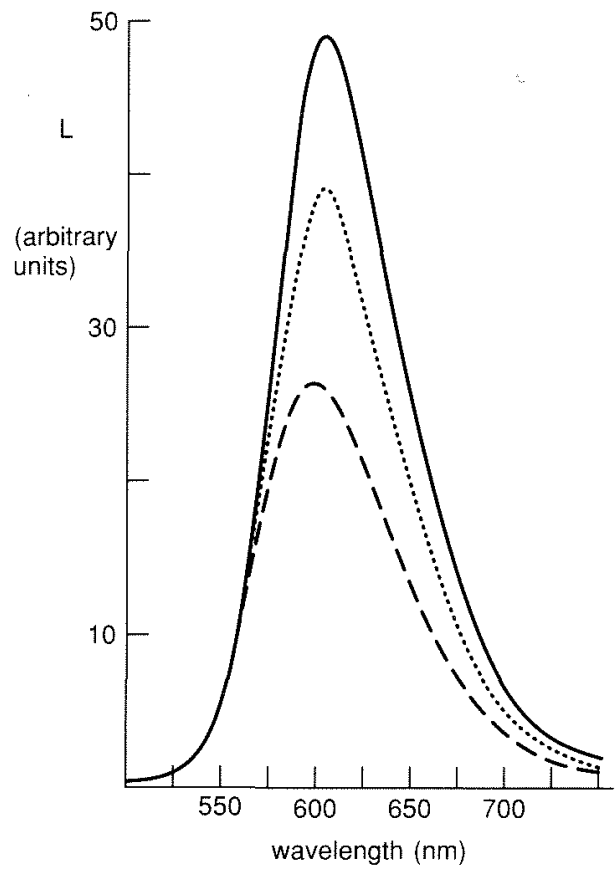

(A)

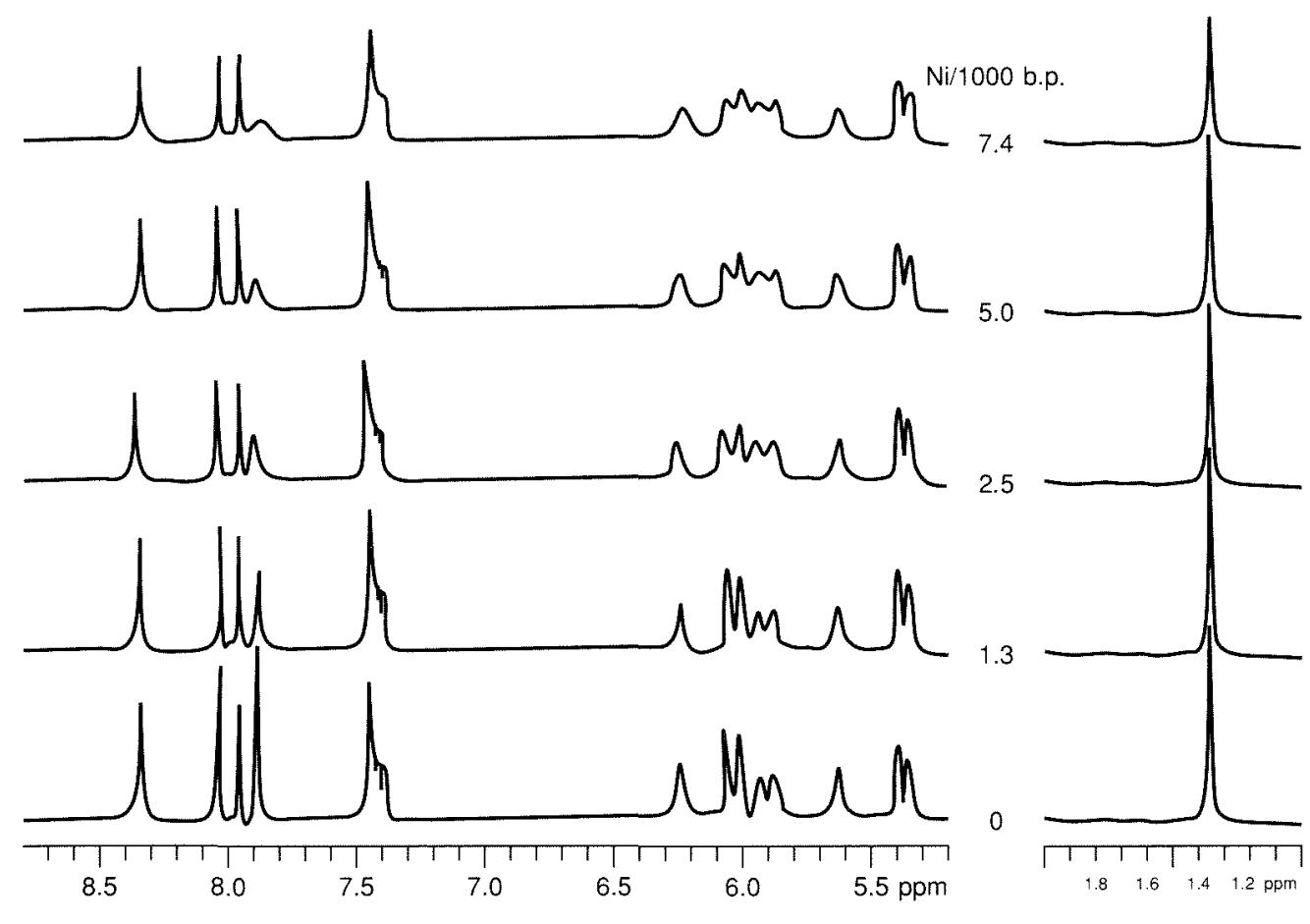

(B) 
There are numerous other classic techniques of inorganic chemistry that have been or could be applied in studying the binding of metal complexes to nucleic acids. Coordination complexes have invariably been used in $\mathrm{x}$-ray diffraction experiments because of the high electron density of the metal center. The tris(phenanthroline) metal complexes have not yet been applied in this context, but, as mentioned already, platinum metallointercalators were examined by fiber diffraction to delineate intercalation requirements. In fact, many nucleic-acid crystal structures have required specific metal ion additions for isomorphous heavy-metal derivatives to solve the structure. Such has certainly been true for the crystal structure of tRNA ${ }^{\text {Phe }}$, where heavy-metal ions such as platinum, osmium, and mercury were targeted to specific base positions, and lanthanide ions were used to label phosphate positions around the periphery of the molecule. ${ }^{53}$ Other techniques can also be exploited to monitor and characterize binding. A recent novel illustration is one from electrochemistry, which has been applied in monitoring the binding of $\mathrm{Co}(\text { phen })_{3}{ }^{3+}$ to DNA. ${ }^{54}$ Surely other techniques, from EXAFS to scanning tunneling microscopy, will be exploited in the future.

Biochemistry also provides very sensitive techniques that have been invaluable in characterizing interactions of metal complexes with nucleic acids. First are simply gel electrophoresis experiments, which permit an assessment of changes in the nucleic-acid conformation, through its changes in gel mobility, as a function of metal binding. A classic illustration is that of the unwinding of superhelical DNA as a function of intercalation. Closed circular DNA has much the same topological constraints on it as does a rope or a telephone cord; the DNA helices can wind up in coils. We define the duplex turning in a double helix as the secondary helical turns, and turns of the helices about one another as the supercoils or tertiary turns. As long as a DNA double helix is closed in a circle (form I), the total winding, that is, the total number of secondary and tertiary turns, is fixed. Molecules with differing extents of winding have different superhelical densities. In a circular molecule with one strand scission, what we call form II (nicked) DNA, the topological constraints are relaxed, and no supercoils are apparent. The same, by analogy, can be said of a telephone cord

\section{Figure 8.9 (facing page)}

An illustration of two spectroscopic techniques used to probe DNA. (A) The variation in luminescence characteristics of $\mathrm{Ru}(\text { phen })_{3}{ }^{2+}$ with DNA binding. Shown is the emission spectrum of free $\mathrm{Ru}(\mathrm{phen})_{3}{ }^{2+}(\ldots-\ldots), \Lambda-\operatorname{Ru}(\mathrm{phen})_{3}{ }^{2+}$ in the presence of DNA $(\cdots \cdots)$, and $\Delta-\mathrm{Ru}(\mathrm{phen})_{3}{ }^{2+}$ in the presence of DNA (-) illustrating the spectroscopic perturbation with DNA binding as well as the associated enantioselectivity in binding of the complexes to the helix. As is evident from the greater luminescence of the $\Delta$ isomer on binding, it is this $\Delta$-isomer that intercalates preferentially into the right-handed helix. (B) An application of paramagnetic broadening by metal complexes in NMR experiments to obtain structural information on their association with nucleic acids. Shown is the ${ }^{1} \mathrm{H}-\mathrm{NMR}$ spectrum of $\mathrm{d}(\mathrm{GTGCAC})_{2}$ with increasing amounts of $\Lambda$-Ni(phen $)_{3}{ }^{2+}$. Note the preferential broadening of the adenine AH2 resonance $(7.8 \mathrm{ppm})$, indicating the association of this enantiomer in the minor groove of the helix. 
off the phone receiver, which can turn about itself to relax its many supercoils. Now let us consider a DNA unwinding experiment, monitored by gel electrophoresis. Supercoiled form I DNA can be distinguished from nicked DNA (form II) in an agarose gel because of their differing mobilities; the wound-up supercoiled molecule moves easily through the gelatinous matrix to the positive pole, whereas the nicked species is more floppy and thus is inhibited in its travels down the gel. A closed circular molecule with no net supercoils ( form $\mathrm{I}_{0}$ ) comigrates with the nicked species. Consider now the supercoiled molecule in the presence of an intercalator. Since the intercalator unwinds the DNA base pairs, the number of secondary helical turns in the DNA is reduced. In a negatively supercoiled, closed circular DNA molecule, the number of supercoils must be increased in a compensatory fashion (the total winding is fixed); hence the total number of negative supercoils is reduced, and the molecule runs with slower mobility through the gel. As the intercalator concentration is increased still further, the mobility of the supercoiled species decreases until no supercoils are left, and the species comigrates with the nicked form II DNA. Increasing the bound intercalator concentration still further leads to the positive supercoiling of the DNA and an increase in mobility. Figure 8.10 illustrates the experiment with tris(phenanthroline)ruthenium(II) isomers. ${ }^{46}$ This kind of unwinding experiment is an example of the sensitivity with which DNA structural changes can be monitored using biochemical methods; only low quantities $(<\mu \mathrm{g})$ of materials are needed to observe these effects.

DNA strand scission can also be sensitively monitored, and even more importantly the specific nucleotide position cleaved can be pinpointed by biochemical methods. This methodology has been applied successfully in monitoring both the efficiency of DNA strand scission by metal complexes and the specific sites cleaved, and hence where the complexes are specifically bound on the helical strand.

Relative extents of cleavage of DNA by different metal complexes can be easily assayed in an experiment that is an extension of the unwinding experiment described above. One simply measures the conversion of supercoiled form I DNA to nicked form II species. One strand cleavage on the DNA circle releases the topological constraints on the circular molecule and relaxes the supercoils. Two cleavage events within 12 base pairs on opposite strands will convert the DNA to a linear form (III), which also has a distinguishable gel mobility. Photoactivated cleavage of DNA by tris(phenanthroline) complexes of cobalt(III) and rhodium(III) was first demonstrated using this assay. ${ }^{55,56}$ Given the high sensitivity of this assay, redox-mediated cleavage of DNA by a wide range of metal complexes can be easily demonstrated. However, other techniques are required to analyze whether appreciable and significant cleavage results, and, if so, what products are obtained. Since the assay can monitor, in a short time using little sample, a single nick in a full 4,000-base-pair plasmid, reactions of very low, almost insignificant yield can be detected. The assay provides, however, a simple scheme to assess relative extents of cleavage by different metal 


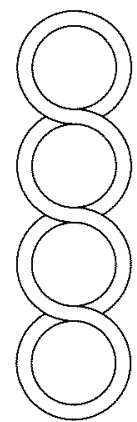

form I

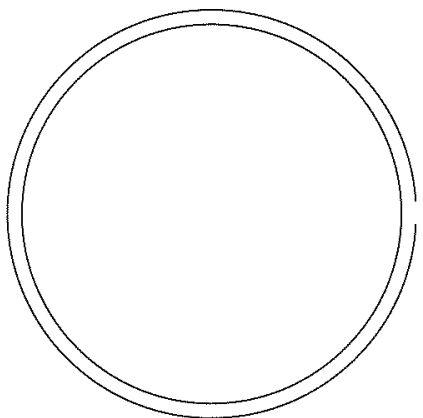

form II form III

(A)
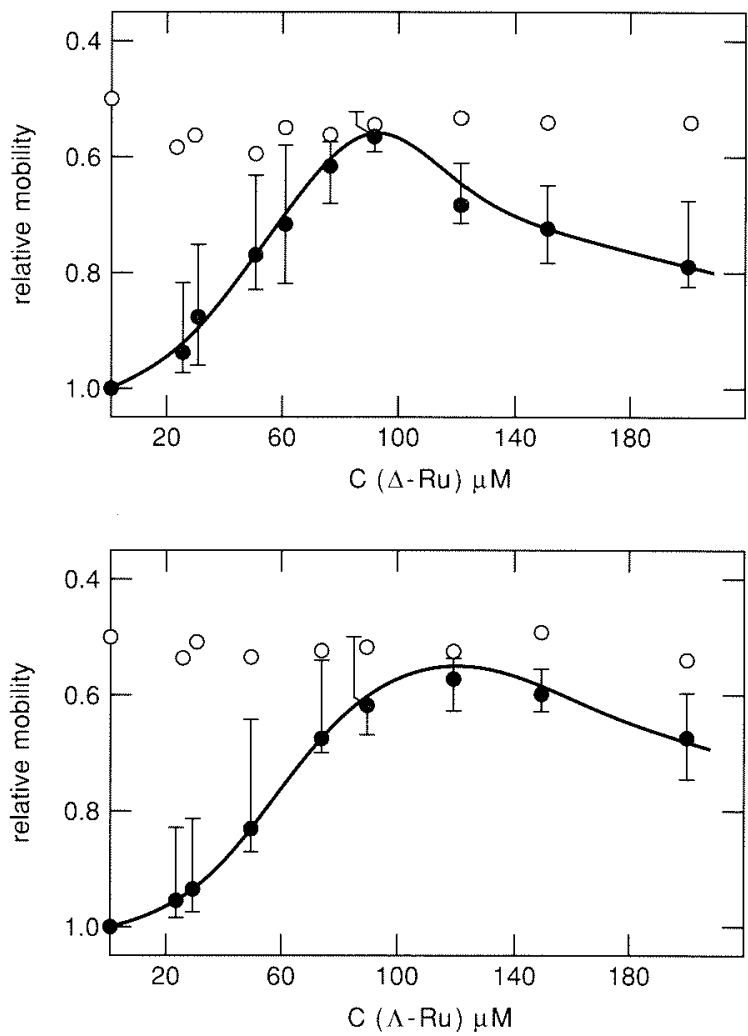

(B)

Figure 8.10

The application of DNA supercoiling to probe metal-complex interactions with DNA. (A) A schematic representation of supercoiled DNA (form I), nicked DNA (form II) that, as a result of the single-strand scission, relaxes to a circular form lacking supercoils, and linear (form III) DNA. (B) Plots of the relative electrophoretic mobilities of form I $(\bullet)$ and form II (O) DNA as a function of increasing concentration of $\Delta$-(top) and $\Lambda$-(bottom) Ru(phen) ${ }_{3}{ }^{2+}$ in the gel. ${ }^{46} \mathrm{In}$ creasing concentrations of bound intercalator unwind the negatively supercoiled DNA. Given the higher intercalative binding affinity of the $\Delta$-isomer, slightly lower concentrations of this isomer are needed to unwind the plasmid to a totally relaxed state (where form I and II comigrate). 
complexes, as well as a first indication that a cleavage reaction by a given metal complex occurs at all.

More informative are experiments on ${ }^{32} \mathrm{P}$-labeled DNA fragments using highresolution polyacrylamide gel electrophoresis, since these experiments allow one to find the exact nucleotide where the complexes break the sugar-phosphate backbone. Consider a cleavage reaction by a given metal complex on a DNA fragment of 100 base pairs in length that has been labeled enzymatically with ${ }^{32} \mathrm{P}$ on one end of one strand. If the metal complex cleaves the DNA at several different sites, then one can arrive at conditions where full cleavage is not obtained, but instead a population of molecules is generated where single cleavage events per strand are obtained, and cleavage at each of the sites is represented. After denaturation of the fragment, electrophoresis through a high-density polyacrylamide gel, and autoradiography, only fragments that are radioactively endlabeled are detected, and hence the population of sites cleaved is determined. The denatured cleaved fragments move through the gel according to their molecular weight. By measuring their length, using molecular-weight markers, one can find the specific position cleaved relative to the end of the fragment. By this route the specific sites cleaved by a molecule that binds and cleaves DNA, or end-labeled RNA, at unique positions may be identified. In a complementary experiment, using footprinting, where a molecule cleaves DNA nonspecifically at all sites along a fragment, one can find the binding positions of other molecules such as DNA-binding proteins. In this experiment, cleavage with the sequence-neutral cleaving reagent is carried out both in the presence and in the absence of the other binding molecule. In the absence of the protein, cleavage ideally occurs at all sites; hence a ladder of cleaved fragments is apparent on the autoradiograph. If cleavage is carried out in the presence of the protein, however, those sites that are bound by the protein are protected from cleavage by steric considerations, producing a shadow or footprint of the protein-binding site on the gel. Both the site-specific and footprinting experiments are illustrated schematically in Figure 8.11. This very powerful technology was first applied by Dervan and coworkers in demonstrating the application of methidium-propylFeEDTA (MPE-Fe) as a chemical footprinting reagent. ${ }^{22,57}$ Tris(phenanthroline) metal complexes have been shown to cleave DNA nonspecifically, and their derivatives have been applied either as sensitive photofootprinting reagents, or as site-specific cleaving molecules, as we will see.

\section{APPLICATIONS OF DIFFERENT METAL COMPLEXES THAT BIND NUCLEIC ACIDS}

Both the spectroscopy and the chemical reactivity of transition-metal complexes, coupled to biochemical assays, can therefore be exploited to obtain a wide range of useful reagents to probe nucleic acids. Here some specific applications are described. 
A: cleavage by a sequence specific metal complex

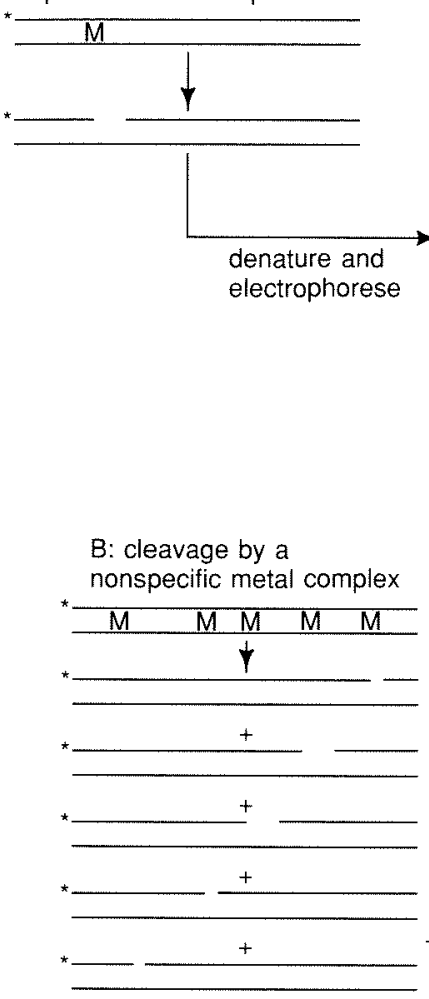

C: cleavage by a nonspecific metal complex in the presence of protein polyacrylamide gel
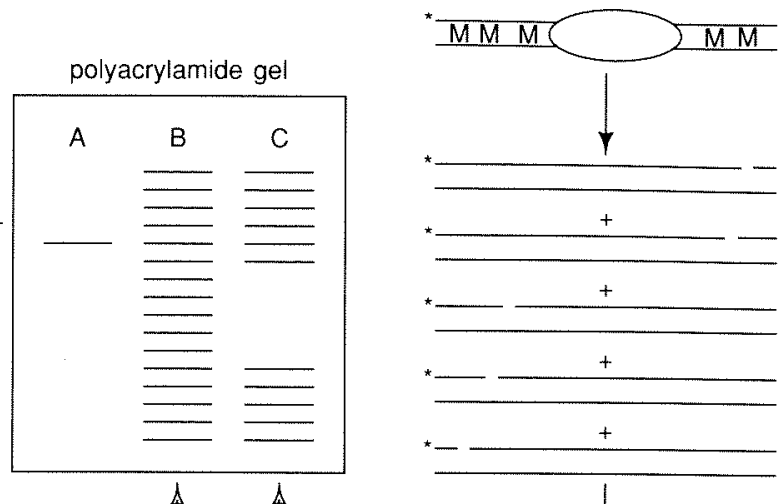

\section{Figure 8.11}

DNA cleavage by metal complexes. Shown schematically is the method used with single-base resolution to discover the sites where metal complexes are bound on double helical DNA. After the metal complex is bound to several sites on a radioactively end-labeled (*) DNA fragment and activated to permit strand cleavage at the binding sites, the nicked DNA is denatured and electrophoresed on a high-resolution polyacrylamide gel, and the gel submitted to autoradiography. From the molecular weights of the end-labeled denatured fragments, the positions of cleavage and therefore binding by the metal complex may be deduced. The results in lane A show the cleavage pattern observed for a metal complex that binds to a specific site. The results in lane $\mathrm{B}$ show cleavage observed for a nonspecifically bound metal complex that binds and therefore cleaves at every base site. Lane $\mathrm{C}$ illustrates a footprinting experiment. When protein is bound to DNA at a specific site, it protects the DNA from cleavage by the metal complex at its binding site, thus producing a "footprint" in the gel: the absence of end-labeled fragments of those lengths that are protected from cleavage as a result of protein binding.

\section{A. Spectroscopic Probes}

As discussed above, the tris(phenanthroline)ruthenium(II) complexes offer a novel spectroscopic probe of nucleic acids, since their luminescence is increased upon intercalation into the double helix. As a result the complexes provide a simple luminescent stain for DNA in fluorescent microscopy experiments. More interesting, perhaps, is the conformational selectivity of derivatives of tris(phenanthro- 
line)ruthenium. $\mathrm{Ru}(\mathrm{DIP})_{3}{ }^{2+}(\mathrm{DIP}=4,7$-diphenyl-1,10-phenanthroline $)$ shows enantiospecificity in binding to B-form DNA. ${ }^{40}$ Because of the steric bulk of the phenyl rings, detectable binding is seen only with the $\Delta$-isomer in a righthanded helix; no binding is evident with the $\Lambda$-isomer. But with the left-handed Z-form helix, both isomers bind avidly. ${ }^{40,58}$ The shallow left-handed major groove can accomodate the two enantiomers. A left-handed but more B-like helix shows selectivity instead for the $\Lambda$-isomer. Spectroscopic experiments that measure the chiral selectivity of $\mathrm{Ru}(\mathrm{DIP})_{3}{ }^{2+}$ isomers in binding to a given DNA then provide a novel probe for helical handedness. Indeed, $\Lambda$-Ru(DIP $)_{3}{ }^{2+}$ was the first spectroscopic probe for Z-DNA (or other alternate conformations that are sufficiently unwound to permit binding by the bulky left-handed isomer). ${ }^{58}$

Another set of derivatives of the tris(phenanthroline) metal complexes that may become exceedingly useful as spectroscopic probes are $\mathrm{Ru}(\mathrm{bpy})_{2} \mathrm{dppz}{ }^{2+}$ and $\mathrm{Ru}(\mathrm{phen})_{2} \mathrm{dppz} \mathrm{z}^{2+}(\mathrm{dppz}=$ dipyridophenazine $){ }^{59}$ In these complexes the metal-to-ligand charge transfer is preferentially to the electron-accepting dppz ligand. In nonaqueous solutions, the complexes luminesce. However, in aqueous solution at $\mathrm{pH} 7$, no luminescence is observed, likely because hydrogen bonding by water to the phenazine nitrogen atoms quenches the charge-transfer excited state. But the dppz ligand is also an expansive, aromatic heterocyclic ligand, and as a result both $\mathrm{Ru}(\mathrm{bpy})_{2} \mathrm{dppz} \mathrm{z}^{2+}$ and $\mathrm{Ru}(\mathrm{phen})_{2} \mathrm{dppz}^{2+}$ bind avidly to DNA by intercalation. Once intercalated, the phenazine ligand is protected from water. Therefore these complexes are luminescent when intercalated into DNA, whereas no luminescence is apparent from the complexes in the absence of DNA in aqueous solution. The enhancement factor is $>10^{4}$ with DNA. One might consider the ruthenium complexes as true "molecular light switches" for DNA.

Both simpler bipyridyl and phenanthroline derivatives as well as dppz complexes of ruthenium are currently being tethered onto other DNA binding moieties, in particular onto oligonucleotides, so as to develop new, nonradioactive luminescent probes for DNA sequences. These transition-metal complexes may provide the basis for the development of new families of DNA diagnostic agents, and many industrial laboratories are currently exploring routes to accomplish these goals. Figure 8.12 illustrates $\Lambda$-Ru(DIP $)_{3}{ }^{2+}$ and $R u(b p y)_{2} d_{p p z}{ }^{2+}$, two complexes whose luminescence properties can be employed to probe nucleic acids.

Other transition-metal complexes besides those of ruthenium have shown some promise in spectroscopic applications with nucleic acids. Lanthanide ions have been applied both in NMR experiments and in luminescence experiments to probe tRNAs, and more recently with synthetic DNAs of differing sequence and structure. ${ }^{60}$ Lanthanide ions have been exceedingly useful in probing $\mathrm{Ca}^{2+}$ binding sites in proteins, and one would hope that a parallel utility would be achieved with nucleic acids. Their poor absorptivity have made luminescent experiments difficult, however, requiring relatively high concentrations of material. Nonetheless, the sensitivity of luminescent lifetimes to coordination and indeed solvation is providing a novel spectroscopic handle to explore binding sites and structures of the macromolecules. Another quite novel luminescent 


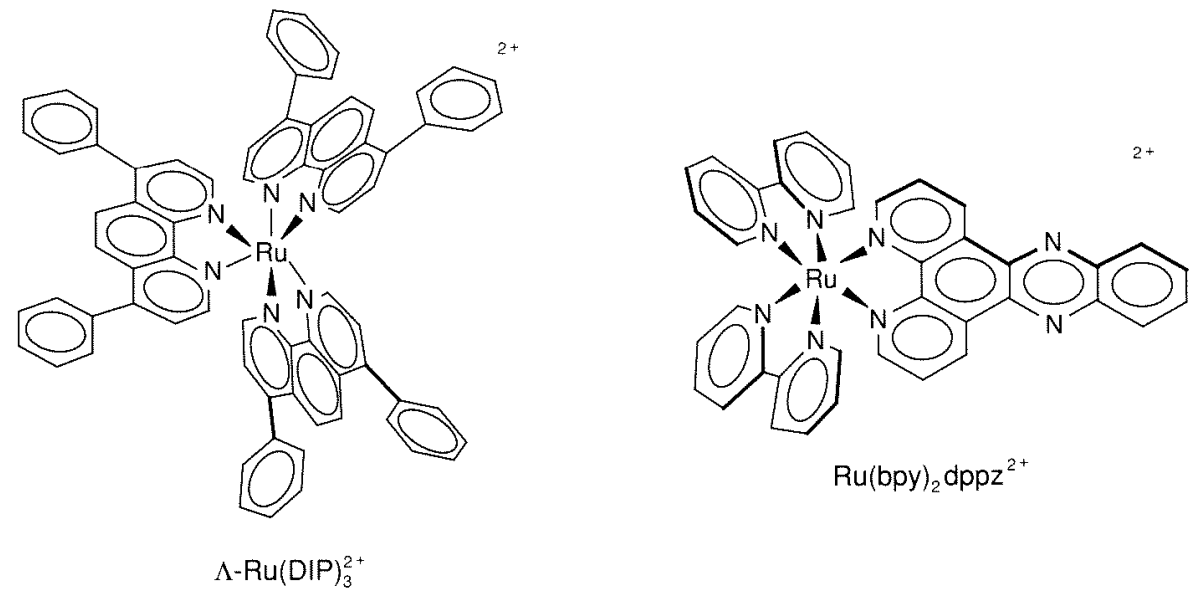

Figure 8.12

Two spectroscopic probes of nucleic acids: $\Lambda-\operatorname{Ru}(\mathrm{DIP})_{3}{ }^{2+}$ and $\mathrm{Ru}(\mathrm{bpy})_{2} \mathrm{dppz}{ }^{2+}$.

handle has been phenanthroline and diphenylphenanthroline complexes of copper(I). ${ }^{61}$ These complexes are extremely valuable cleavage probes, as we will see later; to characterize better their interactions with the helix, luminescence experiments are being explored. A problem here has been the nonphysiological conditions necessary to achieve detectable luminescence. Nonetheless, studies with the copper complexes demonstrate how the whole range of transition-metal chemistry and spectroscopy is beginning to be applied in sorting through nucleic-acid interactions.

\section{B. Metallofootprinting Reagents}

Probably the most widespread application of metal nucleic-acid chemistry in the biology community has been the utilization of metal complexes for chemical footprinting. The footprinting technique (Figure 8.11) was developed by biologists ${ }^{62}$ as a means of locating protein-binding sites on DNA. ${ }^{32} \mathrm{P}$-end-labeled double-stranded DNA fragments could be digested with a nuclease, such as DNAse, in the presence or absence of DNA-binding protein. After electrophoresis of the denatured digests and autoradiography, one would find a "footprint," that is, the inhibition of cleavage by DNAse, at the spot bound by protein, in comparison to a randomly cleaved pattern found on the DNA in the absence of binding protein. Although DNAse is still widely used, this footprinting reagent has some disadvantages: (i) the nuclease is not sequence-neutral in its cleavage, resulting in lots of noise in the footprinting background; and (ii) since the nuclease is itself a large protein, its ability to provide high-resolution footprinting patterns of smaller molecules is quite limited.

Several metal complexes now serve as high-resolution, sequence-neutral chemical footprinting reagents. Some of these reagents are shown in Figure 8.13. The first, as mentioned previously, was MPE-Fe(II). ${ }^{57}$ The complex con- 


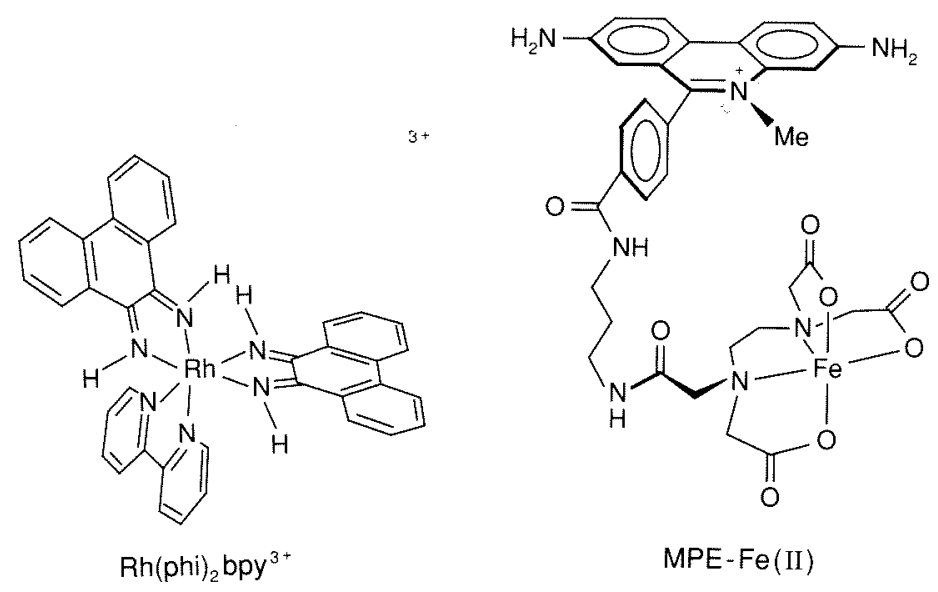

Figure 8.13

Examples of metallofootprinting reagents. $\mathrm{Rh}\left(\mathrm{phi}_{2} \mathrm{bpy}^{3+}\right.$, a photofootprinting intercalator, and MPE-Fe(II), a sequence-neutral intercalating agent.

tains a sequence-neutral DNA binding moiety, the intercalator methidium, and a tethered DNA redox cleaving moiety, Fe(EDTA). The methidium, in binding nonspecifically to DNA, delivers the hydroxyl radicals, generated via Fenton chemistry at the $\mathrm{Fe}(\mathrm{II})$ center in the presence of peroxide and a reducing agent, to the DNA backbone in a random manner. Since the complex is small, high resolution can be achieved. Indeed, MPE-Fe(II) has been shown to footprint small natural products that bind to DNA, in addition to footprinting much larger DNA-binding peptides and proteins.

Perhaps simpler still and now very widely used as a footprinting reagent is $\mathrm{Fe}(\mathrm{EDTA})^{2-}$ itself. $^{63}$ The concept here is that Fe(EDTA) ${ }^{2-}$, as a dianion, is unlikely to associate at all with the DNA polyanion. Hence hydroxyl radicals, generated via Fenton chemistry at a distance from the helix, would likely diffuse to the helix with a uniform concentration along the helix and provide a completely sequence-neutral pattern of cleavage. Tullius and coworkers have demonstrated ${ }^{63}$ this to be the case. The resolution is furthermore extremely high since the hydroxyl radical is sufficiently small that it can even diffuse within the DNA-binding protein to delineate binding domains. Some difficulties are found, however, with the high concentrations of activating reagents needed to activate a cleavage reagent that does not bind to the helix, and problems of course arise in trying to footprint metalloproteins. Nonetheless, Fe(EDTA) ${ }^{2-}$, a reagent easily found on the biologist's shelf, is now finding great utility in labs as a chemical substitute for DNAse.

Other transition-metal complexes are also finding applications in chemical footprinting. Both $\mathrm{Cu}$ (phen) ${ }_{2}{ }^{+}$and manganese porphyrins have been used to footprint DNA-binding proteins. ${ }^{64,65}$ These complexes likely cleave DNA through 
either Fenton chemistry or direct reaction of a coordinated metal-oxo intermediate with the sugar-phosphate backbone. The complexes, however, appear to bind DNA predominantly along the surface of the DNA minor groove, and with some preference for AT-rich regions. The patterns obtained are actually quite similar to those found with DNAse, and thus the lack of high sequence neutrality is somewhat limiting. Furthermore, the complexes are most sensitive to binding moieties in the minor groove, rather than those in the major groove, where proteins bind. Intercalators such as MPE-Fe(II) can sense binding species in both grooves. $\mathrm{Cu}$ (phen $)_{2}{ }^{+}$has nonetheless proved to be quite effective in detecting hyperreactivities in the minor groove, owing to DNA structural perturbations that arise from protein binding in the major groove. Whether this sensitivity emanates from the intimate interaction of $\mathrm{Cu}(\mathrm{phen})_{2}{ }^{+}$in the minor groove of the helix, or because of the characteristics of the reactive radical formed, is not known.

Inorganic photochemistry has also been applied in developing metal complexes as photofootprinting reagents. Uranyl acetate, for example, at high concentrations, upon photolysis, promotes DNA cleavage. ${ }^{66}$ It is thought that the ions interact with the phosphates, generating some excited-state radical chemistry, although no detailed characterization of this chemistry has been undertaken. The cleavage reaction is nonetheless remarkably sequence-neutral and therefore shows some promise for photofootprinting applications. In fact, the applicability of uranyl acetate typifies how simple coordination chemistry and now even photochemistry may be helpful in the design of a variety of reagents that interact and cleave DNA, both nonspecifically and specifically. The biochemical techniques used to monitor such processes are sufficiently sensitive that even quite inefficient reactions in solution can be harnessed in developing useful reagents. The better our understanding of the chemistry of the coordination complex, the more effectively it may be utilized.

The best derivative of a tris(phenanthroline) metal complex currently being applied in footprinting experiments is $\mathrm{Rh}(\mathrm{phi})_{2} \mathrm{bpy}^{3+}$, a second-generation derivative of the tris(phenanthroline) $\operatorname{series}^{67}$ that binds DNA avidly by intercalation and in the presence of light promotes direct strand cleavage by hydrogenatom abstraction at the $\mathrm{C} 3{ }^{\prime}$-position on the sugar. ${ }^{31}$ Since no diffusing intermediate is involved in this photocleavage reaction, the resolution of the footprinting pattern is to a single nucleotide. Here the excited-state transition-metal chemistry involves a ligand-to-metal charge transfer, producing a phi cation radical that directly abstracts the hydrogen from the sugar at the intercalated site. The high efficiency of this photoreaction and high sequence-neutral binding of the complex to double-stranded DNA add to the utility of this reagent in footprinting studies. Indeed, both DNA-binding proteins, bound in the major groove, and small natural products, associated with the minor groove, have been footprinted with $\mathrm{Rh}(\mathrm{phi})_{2} \mathrm{bpy}^{3+}$ to precisely that size expected based upon crystallographic results. One may hope that this and other photofootprinting reagents will soon find applications for footprinting experiments in vivo. 


\section{Conformational Probes}

Metal complexes are also finding wide application in probing the local variations in conformation that arise along nucleic-acid polymers. X-ray crystallography has been critical in establishing the basic conformational families of doublehelical DNA, and to some extent how conformations might vary as a function of nucleic-acid sequence. Yet many conformations have still not been described to high resolution, and only a few oligonucleotides have been crystallized. Other techniques are therefore required to bridge the small set of oligonucleotide crystal structures that point to plausible structures and the large array of structures that arise as a function of sequence on long helical polymers. Furthermore, only a very small number of RNA polymers has been characterized crystallographically; hence other chemical methods have been needed to describe the folding patterns in these important biopolymers. Metal complexes, mainly through specific noncovalent interactions, appear to be uniquely useful in probing the structural variations in nucleic acids.

\section{Nonspecific reactions of transition-metal complexes}

Hydroxyl radical cleavage with $\mathrm{Fe}(\mathrm{EDTA})^{2-}$ illustrates again how simple metal complexes can be used in characterizing nucleic acids. One example involves efforts to describe the local structural variations in "bent" DNA. Biochemists had found that DNA fragments containing runs of adenines, such as in the tract dAAAAAA, possessed unusual gel-electrophoretic mobilities. Indeed, kinetoplast DNA isolated from mitochondria of trypanosomes showed a remarkable lacework pattern of structure, with loops and circles of DNA; these structures were found to be governed by the placement of these $d(A)_{6}$ tracts. By constructing a series of oligonucleotides with adenine runs positioned either in or out of phase relative to one another, researchers found that the adenine tracts caused a local bending of the DNA toward the minor groove. ${ }^{6}$ But what were the detailed characteristics of these bent sites? Using hydroxyl radical cleavage of DNA, generated with Fe(EDTA) ${ }^{2-}$, Tullius and coworkers found a distinctive pattern of cleavage across the adenine tracts, consistent with a locally perturbed structure. ${ }^{68}$ Here the notion again was that Fe(EDTA) ${ }^{2-}$ in the presence of peroxide would generate hydroxyl radicals at a distance from the helix, and thus careful densitometric analysis of the cleavage across ${ }^{32} \mathrm{P}$-end-labeled DNA fragments would reveal any differential accessibility of sugar residues to cleavage mediated by the radicals caused by the bending. The cleavage patterns suggested a smooth bending of the DNA across the tract and indicated furthermore an asymmetry in structure from the $5^{\prime}$ - to $3^{\prime}$-end of the adenine run.

The reactivities of other transition-metal reagents have also been used advantageously in probing nucleic-acid structures. As described in Section II, $\mathrm{OsO}_{4}$ reacts across the 5,6 position of accessible pyrimidines to form a cis-osmate ester. Upon treatment with piperidine, this base modification can yield scission of the sugar-phosphate backbone. Hence DNAs containing unusual local con- 
formations with prominent solvent-accessible pyrimidines can be probed with $\mathrm{OsO}_{4}$. The junction regions of Z-DNA, the single-stranded loops in cruciform structures, and a segment of the dangling third strand in H-DNA, have all been probed by means of the differential reactivity of osmium tetroxide with DNA sites dependent upon their accessibility. ${ }^{7,8,16,69}$ Surely other transition-metal chemistry will become similarly applicable.

\section{Transition-metal complexes as shape-selective probes}

Transition-metal complexes have also been designed with three-dimensional structures that target complementary structures along the helical polymer. This recognition of DNA sites, based upon shape selection, has proved to be extremely useful both in demarcating and in characterizing structural variations along the polymer and in developing an understanding of those factors important to the recognition of specific polynucleotide sites. Complexes, basically derivatives of the tris(phenanthroline) metal series, have been designed that specifically target A- and Z-form helices, cruciforms, and even subtle variations such as differential propeller twisting within B-form DNA. ${ }^{11 \mathrm{c}}$ By appropriate substitution of the metal at the center of the coordinatively saturated complex, complexes that cleave the DNA at the binding site are obtained. Figure 8.14 shows some of these shape-selective conformational probes.

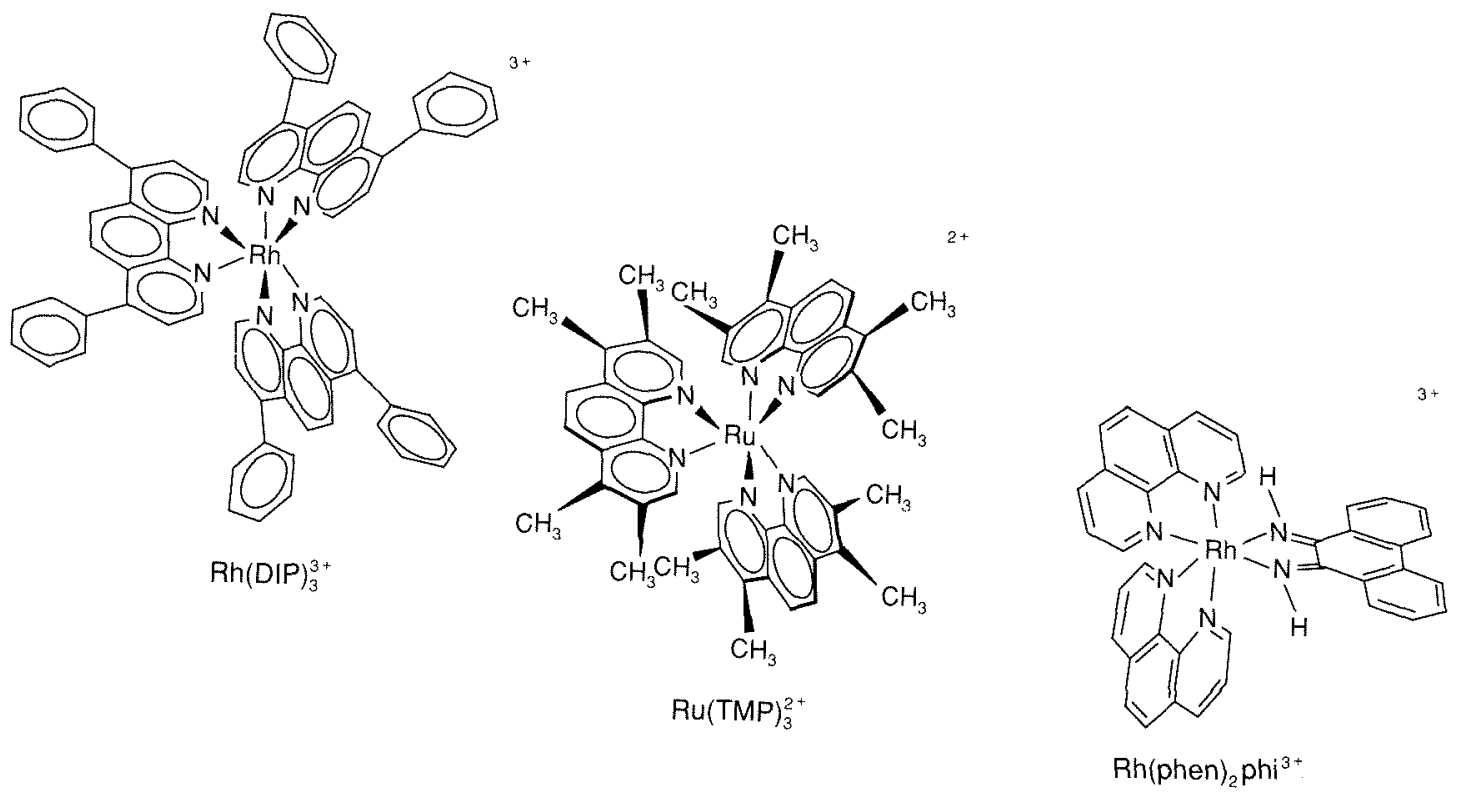

Figure 8.14

Shape-selective probes that target local DNA conformations. Rh(DIP $)_{3}{ }^{3+}$, which with photoactivation promotes double-stranded cleavage at cruciform sites; $\mathrm{Ru}(\mathrm{TMP})_{3}{ }^{2+}$, a photoactivated probe for A-like conformations; and $\mathrm{Rh}(\mathrm{phen})_{2} \mathrm{phi}^{3+}$, which targets openings in the DNA major groove. 
One example of this shape-selective cleavage is apparent in reactions of $\mathrm{Ru}(\mathrm{TMP})_{3}{ }^{2+} \quad(\mathrm{TMP}=3,4,7,8$-tetramethylphenanthroline $)$, a probe of the A-conformation. ${ }^{28,29}$ The complex was designed by incorporating methyl groups about the periphery of each phenanthroline ligand to preclude intercalative binding of the complex to the helix, owing to the bulkiness of the methyl groups, and at the same time to promote hydrophobic groove binding. Importantly, however, this hydrophobic groove binding could not occur against the minor groove of B-DNA, given the width and depth of the groove versus the size of the complex. Instead, the shape of the complex was matched well to the shallow minor-groove surface of an A-form helix. Binding studies with synthetic polynucleotides of A, B, and Z-form were consistent with this scheme. Photolysis of the ruthenium complex, furthermore, as with $\mathrm{Ru}(\mathrm{phen})_{3}{ }^{2+}$, leads to the sensitization of singlet oxygen, and hence, after treatment with piperidine, to strand cleavage. Thus, photocleavage reactions with $\mathrm{Ru}(\mathrm{TMP})_{3}{ }^{2+}$ could be used to delineate A-like regions, with more shallow minor grooves, along a helical polymer. At such sites, Ru(TMP) ${ }_{3}{ }^{2+}$ would bind preferentially, and upon photolysis, generate locally higher concentrations of singlet oxygen to mediate cleavage of the sugar-phosphate backbone. This scheme revealed that homopyrimidine stretches along the helix adopt a more A-like conformation. ${ }^{29}$

The targeting of altered conformations such as Z-DNA has been described earlier ${ }^{58}$ in the context of a spectroscopic probe, $\Lambda-\operatorname{Ru}(\mathrm{DIP})_{3}{ }^{2+}$. Substitution of a photoredox-active metal into the core of the tris(diphenylphenanthroline) unit leads also to a complex that both binds and, with photoactivation, cleaves at the altered conformation. ${ }^{55}$ Both $\mathrm{Co}$ (III) polypyridyl and Rh(III) polypyridyl complexes have been shown to be potent photooxidants. Coupled to site-specific DNA binding, these metal complexes, with photoactivation, become conformationally selective DNA cleavage agents. $\mathrm{Co}(\mathrm{DIP})_{3}{ }^{3+}$, for example, has been shown to cleave specifically at Z-form segments inserted into DNA plasmids. ${ }^{55,70}$ Perhaps even more interesting, on both natural plasmids and viral DNAs, the various sites cleaved by $\operatorname{Co}(\mathrm{DIP})_{3}{ }^{3+}$, corresponding both to Z-form sites and to other locally altered non-B-conformations, coincide with functionally important regions of the genome, e.g., regulatory sites, gene termination sites, and intron-exon joints. ${ }^{70,71}$ The altered structures recognized by the metal complexes, therefore, appear to mark biologically important sites, those presumably recognized also by cellular proteins. Cleavage studies with these metal complexes, therefore, are providing some insight also into how Nature specifically targets and accesses the sequence information encoded along the DNA polymer, sequence information encoded indirectly through local structure.

The most striking example of the specificity to be derived from shape-selective targeting has been given by the double-stranded cleavage induced by $\mathrm{Rh}(\mathrm{DIP})_{3}{ }^{3+}$ at cruciforms. ${ }^{72} \mathrm{Rh}(\mathrm{DIP})_{3}{ }^{3+}$, like its $\mathrm{Co}(\mathrm{III})$ and $\mathrm{Ru}(\mathrm{II})$ congeners, binds to locally unwound, non-B-conformations such as Z-DNA, but interestingly this potent photooxidant yields the specific cleavage of both DNA strands 
at cruciform sites. Lacking any crystallographic information, our understanding of the local structure of a cruciform is poor. In these palindromic sites, a torsionally strained DNA extrudes two intrastrand hydrogen-bonded helices from the main helix (see Figure 8.2B). Clearly the structure is grossly altered and locally unwound. $\operatorname{Rh}(\mathrm{DIP})_{3}{ }^{3+}$ appears to bind into a pocket generated by the folding of the extruded helix onto the main helix. The recognition is of this intricately folded structure, not of the sequence used to generate the cruciform. Studies with the transition-metal complex on different cruciforms should be useful in helping to characterize this interesting tertiary DNA structure.

Shape-selective transition-metal probes have also been useful in delineating more subtle variations in structure, such as the propeller twisting and tilting evident in B-form DNA. ${ }^{30,73} \mathrm{Rh}$ (phen) ${ }_{2} \mathrm{phi}^{3+}$ was found to target preferentially sites in the major groove where the DNA base pairs are more open; this preferential recognition arises from the steric constraints at more-closed intercalation sites because of the bulkiness of the ancillary phenanthroline ligands above and below the intercalation plane. Two straightforward structural perturbations that lead to an opening of the major groove involve the propeller twisting of bases with respect to one another and the tilting of base pairs along the helix. Chiral discrimination in cleavage by $\mathrm{Rh}$ (phen) ${ }_{2} \mathrm{phi}^{3+}$ is now being used quantitatively to discriminate among these structural parameters. The goal in these studies is to use cleavage results with the shape-selective metal complexes to describe the three-dimensional structure of a long double-helical DNA sequence in solution. Probing structurally well-defined sequences with a whole family of shape-selective metal complexes may provide a route to this goal.

As mentioned above, describing the three-dimensional structures of RNAs is an even more complicated task than it is for double-stranded DNAs. Only a few tRNAs have been characterized crystallographically to high resolution, and for other larger RNA structures, such as 5S RNA, or any of the catalytic intervening-sequence RNAs, little is known about their folding characteristics. To understand the regulation and catalytic functions of these biopolymers, we need to develop chemical tools to explore these structures. Figure 8.15 shows the results of cleavage studies using the variety of transition-metal probes on $\mathrm{RNA}^{\text {Phe }}$. Hydroxyl-radical cleavage mediated by $\mathrm{Fe}(\mathrm{EDTA})^{2-}$ reveals the protection of solvent-inaccessible regions, the "inside" of the molecule. ${ }^{23} \mathrm{MPE}-\mathrm{Fe}$ (II) appears to demarcate the double-helical regions, ${ }^{74} \mathrm{Cu}(\text { phen })_{2}{ }^{+}$shows the loopedout single-stranded segments, ${ }^{75}$ and $\mathrm{Rh}(\mathrm{phen})_{2} \mathrm{phi}^{3+}$ seems to delineate those regions involved in triple-base interactions, the sites of tertiary folding. ${ }^{76}$ Taken together, the full structure of the tRNA can be described based upon cleavage data with transition-metal complexes. It therefore seems as if this full family of coordination complexes might be generally useful in delineating RNA structures. Still more work is needed quantitatively to compare the patterns obtained with the few well-characterized structures. Nonetheless, an important role for these and possibly other transition-metal reagents is indicated. 


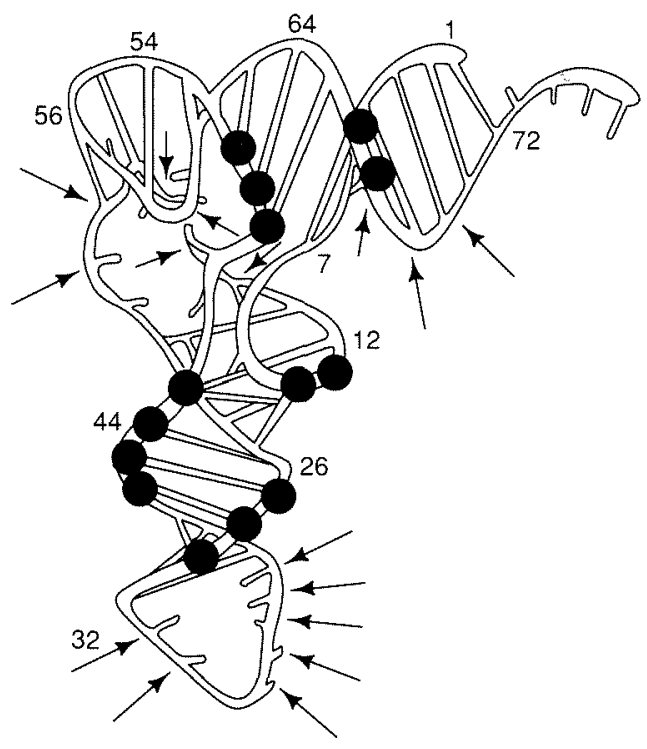

(A)

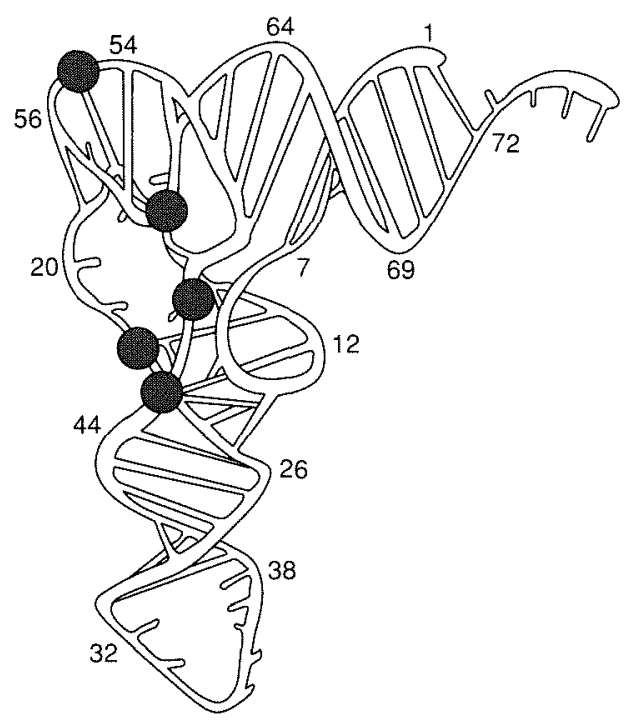

(B)

Figure 8.15

The diversity of cleavage sites for metal complexes on tRNA ${ }^{\text {Phe }}$. In (A) is shown cleavage by probes that primarily detect features of RNA secondary structure. $\mathrm{Cu}(\mathrm{phen})_{2}{ }^{+}$(arrows), detecting single-stranded regions and MPE-Fe(II) (black dots), detecting double-helical segments. In (B) are shown probes that detect protected or more complex structures on tRNA. Inaccessible sites protected from $\mathrm{OH} \cdot$ after treatment with $\mathrm{Fe}(\mathrm{EDTA})^{2-}$ are shown as shaded portions of the molecule, and specific cleavage by $\mathrm{Rh}(\mathrm{phen})_{2}(\mathrm{phi})^{3+}$ at tertiary folds is indicated by the circles. ${ }^{11 \mathrm{~b}}$ 


\section{Other Novel Techniques}

Transition-metal ions can also be used advantageously tethered onto peptides, proteins, oligonucleotides, and other natural products, to provide a chemical probe for their binding interactions with nucleic acids. This strategy, termed affinity cleavage, was developed by Dervan and coworkers in preparing and characterizing distamycin-Fe(II)EDTA. ${ }^{24}$ Distamycin is a known natural product that binds in the minor groove of DNA at AT-rich sequences. By tethering Fe(II)EDTA onto distamycin, the researchers converted the DNA-binding moiety into a DNA-cleaving moiety, since, as with MPE-Fe(II), in the presence of peroxide and a reductant, hydroxyl radical chemistry would be delivered to the distamycin binding site. Unlike MPE-Fe(II), however, the distamycin moiety shows preferential binding at some sites along the polymer, and hence only at those sites would the local hydroxyl-radical concentration be increased and cleavage be obtained. As a result the tethered $\mathrm{Fe}(\mathrm{EDTA})^{2-}$ could be used as a cleavage probe, marking sites of specific binding.

Affinity cleaving has been generalized so that now Fe(EDTA) ${ }^{2-}$ can be tethered onto both oligonucleotides and peptides to follow their interactions with nucleic acids. The sequence-specific binding of oligonucleotides to double-helical DNA through triple-helix formation is but one of many examples where the tethering of $\mathrm{Fe}(\mathrm{EDTA})^{2-}$ has been applied advantageously. ${ }^{77}$

Other redox-active metals can be incorporated into DNA-binding moieties as well. Schemes have been developed to functionalize accessible lysine residues on DNA-binding repressor proteins with phenanthrolines, so that in the presence of copper ion, peroxide, and a reductant, the phenanthroline-bound copper on the protein would induce DNA strand cleavage. Through this scheme, again the conversion of a DNA-binding moiety into a cleaving moiety by incorporation of a redox-active metal, the specific binding sites of repressor proteins can be readily identified (far more quickly on large DNA than through footprinting). ${ }^{78}$

Another scheme, which perhaps takes advantage more directly of bioinorganic chemistry, involves engineering redox metal-binding sites into DNA-binding proteins and peptides. The DNA-binding domain of the protein Hin recombinase was synthesized chemically, and first, to examine the folding of the peptide on the DNA helix, EDTA was tethered onto the peptide for Fe(II) cleavage experiments. ${ }^{79}$ But as is illustrated repeatedly in these chapters, Nature has already provided amino-acid residues for the chelation of metal ions into proteins. Thus the DNA-binding domain of Hin recombinase was synthesized again, now including at its terminus the residues Gly-Gly-His, a known chelating moiety for copper(II). ${ }^{80}$ This chemically synthesized peptide, with now both DNA-binding and DNA-cleaving domains, as illustrated in Figure 8.16, specifically promotes cleavage at the Hin recombinase binding site in the presence of bound copper and ascorbate. Interestingly, the addition of nickel(II) also leads to specific strand cleavage, without diffusible intermediates. Using this approach, taking advan- 


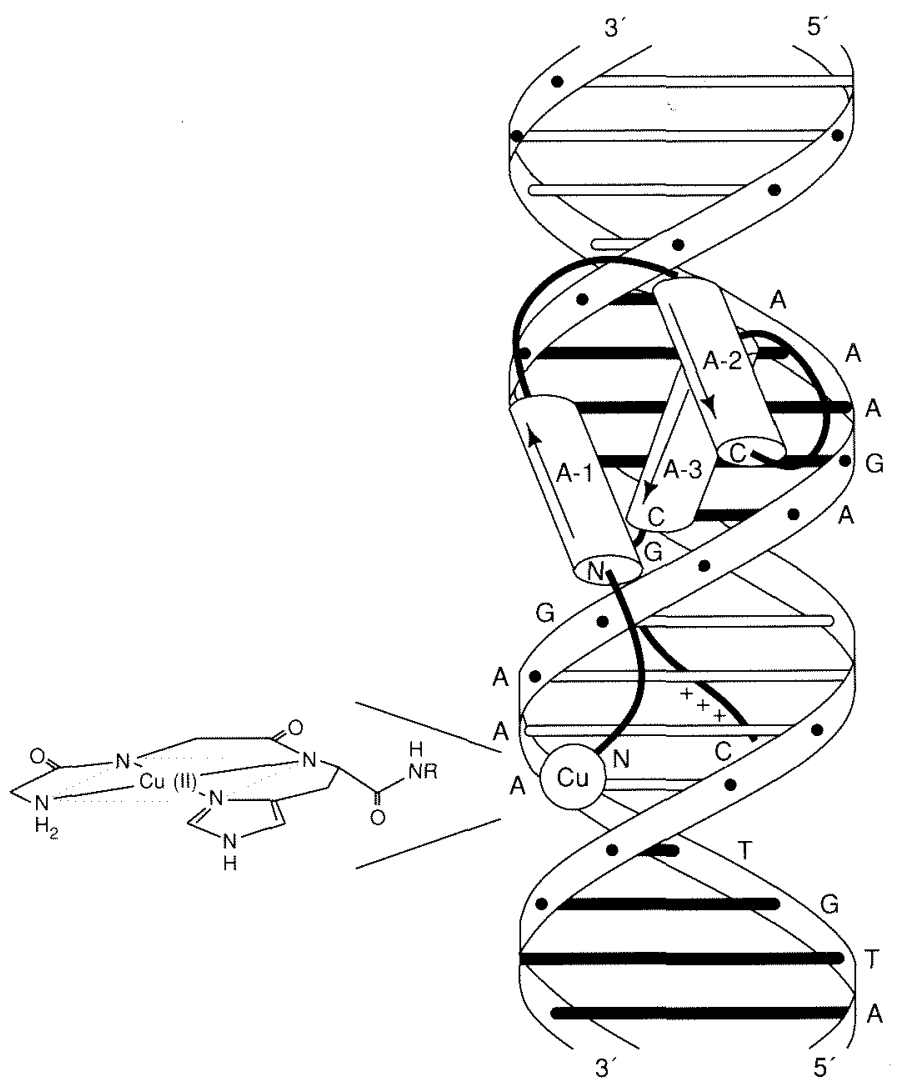

Figure 8.16

A schematic of a synthetic DNA-cleaving peptide bound to DNA that was constructed by synthesis of the DNA-binding domain of Hin Recombinase with Gly-Gly-His at the N-terminus to coordinate copper. ${ }^{80}$ Reproduced with permission from Reference 80 .

tage of the chelating abilities of amino acids and the cleaving abilities of different metal ions, one may prepare new synthetic, functional metalloproteins that bind and react with DNA.

\section{NATURE'S USE OF METAL/NUCLEIC-ACID INTERACTIONS}

In the context of what we understand about the fundamental interactions and reactions of metal ions and complexes with nucleic acids, and also in comparison to how chemists have been exploiting these interactions in probing nucleic acids, we can also consider how Nature has taken advantage of metal ions in the construction of metalloproteins, nucleic-acid assemblies, and smaller natural products containing metal ions that interact with DNA and RNA. 


\section{A. A Structural Role}

One of the chief functions attributed to metal ions in biological systems is their ability to provide a structural center to direct the folding of a protein. Just as shape-selective recognition has been helpful in targeting metal complexes to specific sites on DNA, it appears that one element of the recognition of sites by DNA regulatory proteins may also involve the recognition of complementary shapes. Furthermore, metal ions appear to be used in these proteins to define the shape or folding pattern of the peptide domain that interacts specifically with the nucleic acid.

The DNA-binding metalloproteins that have received the greatest attention recently have been the "zinc-finger" regulatory proteins. It was discovered in 1983 that zinc ions played a role in the functioning of the nucleic acid-binding transcription factor IIIA (TFIIIA) from Xenopus laevis, which binds specifically both DNA, the internal control region of the 5S rRNA gene, and RNA, the 5S RNA itself. ${ }^{81}$ The protein (actually the $7 \mathrm{~S}$ storage particle) was found to contain two to three equivalents of zinc ion. Dialysis removed both the associated zinc ions and the nucleic-acid-binding ability of the protein. Importantly, treatment with zinc ion, or in later studies with higher concentrations of $\mathrm{Co}^{2+}$, restored the specific binding ability. Hence, zinc ion was shown to be functionally important in these eukaryotic regulatory proteins.

The notion of a "zinc-finger structural domain"' was first provided by Klug and coworkers, after examination of the amino-acid sequence in TFIIIA. ${ }^{82}$ It was found that TFIIIA contained nine imperfect repeats of a sequence of approximately 30 amino acids, and furthermore that each repeat contained two cysteine residues, two histidine residues, and three hydrophobic residues, in conserved positions. In addition, subsequent metal analyses were revealing higher zinc contents ( 7 to 11 equivalents) associated with the protein, and proteindigestion experiments indicated that several repeated structural domains existed in the protein. The two cysteine thiolates and two histidine imidazoles in each repeated domain could certainly serve to coordinate a zinc ion. Thus it was proposed that each peptide repeat formed an independent nucleic-acid-binding domain, stabilized in its folded structure through coordination of a zinc ion. The peptide unit was termed a "zinc finger," which is illustrated schematically in Figure 8.17. TFIIIA was therefore proposed to contain nine zinc fingers, which would cooperatively bind in the internal control region of the 5S RNA gene.

An enormous number of gene sequences from a variety of eukaryotic regulatory proteins was then found to encode strikingly similar amino-acid sequences, ${ }^{83}$ and many were dubbed zinc-finger proteins. The bioinorganic chemist, however, should be aware that chemical analyses supporting such assignments are first required. Nonetheless, several legitimate examples of eukaryotic nucleic-acid-binding zinc-finger proteins containing multiple zinc-binding peptide domains have emerged since the first study of TFIIIA, including the proteins Xfin from Xenopus, the Kruppel protein from Drosophila, the Sp1 transcription factor, and human testes-determining factor. It has therefore become clear that 


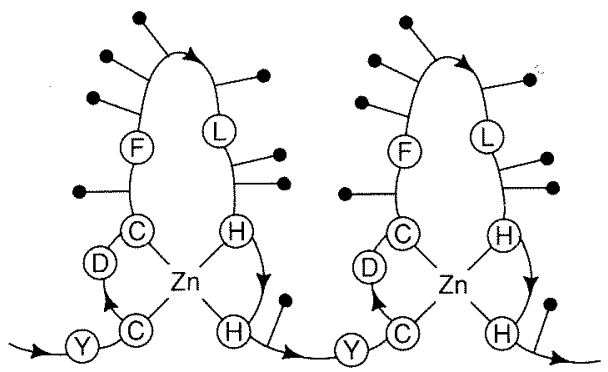

(A)

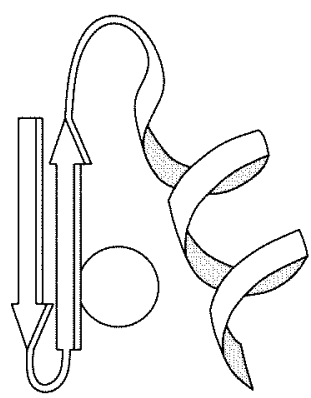

(B)

Figure 8.17

(A) A schematic of a zinc-finger peptide domain. ${ }^{82}$ (B) The proposed schematic structure of a zinc-finger domain based on comparisons to other structurally characterized metalloproteins. ${ }^{85}$

the zinc-finger domain represents a ubiquitous structural motif for eukaryotic DNA-binding proteins. ${ }^{84}$

What is the structure of a zinc finger, and how is this structure important for binding a specific nucleic-acid site? Based on a search of crystallographic databases for metalloproteins and an examination of the consensus sequence emerging for zinc fingers (that is, which residues were truly conserved and common to the different putative zinc fingers), Berg proposed a three-dimensional structure for a zinc finger, shown schematically in Figure $8.17 .^{85}$ The proposed structure included the tetrahedral coordination of zinc by the two cysteine and histidine residues at the base of the finger and an $\alpha$-helical region running almost the length of the domain. EXAFS studies also supported the tetrahedral zinc site. Since this proposal, two detailed two-dimensional NMR studies have been reported that are consistent with the tetrahedral zinc coordination and the $\alpha$-helical segment. ${ }^{86}$ More recently, a crystal structure of a three-finger binding domain associated with an oligonucleotide was determined. ${ }^{87}$ The zinc fingers lie in the major groove of DNA, the $\alpha$-helical region being within the groove. Not surprisingly, given basic coordination chemistry, the zinc does not interact 
directly with the nucleic acid. Instead, the zinc ion must serve a structural role, defining the folding and three-dimensional structure of the protein scaffolding about it. This structure, defined by the metal at its center, like other coordination complexes, is able to recognize its complementary structure on the nucleicacid polymer.

It should also be noted that this zinc-finger structural motif is not the only metal-containing or even zinc-containing structural motif important in nucleicacid-binding proteins. ${ }^{88} \mathrm{~A}$ clearly different domain is evident in the protein GAL4, a transcription factor required for galactose utilization in $S$. cerevisiae ${ }^{88 \text { a }}$ A recent crystal structure of the protein bound to an oligonucleotide shows the protein to bind to DNA as a dimer; each monomer contains a binuclear zinc cluster with two zinc ions tetrahedrally coordinated by six cysteines (two cysteines are bridging), not dissimilar from proposed structures in metallothionein. Still another structural motif is found in the glucocorticoid receptor DNA-binding domain. Crystallography ${ }^{89}$ has revealed that this domain also binds DNA as a dimer; here each monomer contains two zinc-nucleated substructures of distinct conformation. The zinc ions are each tetrahedrally coordinated to four cysteine residues. Likely this too represents another structural motif for proteins that bind nucleic acids, and one again in which the metal serves a structural role.

Lastly, one might consider why zinc ion has been used by Nature in these nucleic-acid binding proteins. Certainly, the natural abundance of zinc is an important criterion. But also important is the absence of any redox activity associated with the metal ion, activity that could promote DNA damage [as with $\mathrm{Fe}(\mathrm{II})$ or $\mathrm{Cu}(\mathrm{II})$, for example]. In addition, other softer, heavier metal ions might bind preferentially to the DNA bases, promoting sequence-specific covalent interactions. Zinc ion, therefore, is clearly well-chosen for the structural center of these various nucleic-acid-binding proteins.

\section{B. A Regulatory Role}

Metalloregulatory proteins, like the transcription factors described above, affect the expression of genetic information through structural interactions that depend upon the metal ions, but unlike the zinc-finger proteins, metalloregulatory proteins act as triggers, repressing or activating transcription given the presence or absence of metal ion. In some respects, even more than zinc fingers, these systems resemble the $\mathrm{Ca}^{2+}$-activated proteins described in Chapter 3 .

Consider the biological system that must respond to changing intracellular metal concentrations. At high concentrations many metal ions become toxic to the cell; hence, a full system of proteins must be synthesized that will chelate and detoxify the bound-metal-ion pool. In order to actively engage these proteins, the genes that encode them must be rapidly transcribed. But at the same time, the DNA itself must be protected from the high concentrations of metal ion. Hence the need for these metalloregulatory proteins, which bind DNA in the absence of metal ion, usually repressing transcription, but in the presence of 
metal ion bind the metal ion tightly and specifically, and as a consequence amplify transcription.

Perhaps the best-characterized metalloregulatory system thus far is the MerR system, regulating mercury resistance in bacteria. ${ }^{90}$ An inducible set of genes arranged in a single operon is under the control of the metal-sensing MerR protein, and it is this system that mediates mercury resistance. Mercury resistance depends upon the expression of these genes to import toxic $\mathrm{Hg}(\mathrm{II})$, reduce $\mathrm{Hg}(\mathrm{II})$ to the volatile $\mathrm{Hg}(0)$ by $\mathrm{NADPH}$, and often additionally to cleave organomercurials to their corresponding hydrocarbon and $\mathrm{Hg}(\mathrm{II})$ species. The MerR protein regulates mercury resistance both negatively and positively. As illustrated in Figure 8.18, MerR in the absence of $\mathrm{Hg}$ (II) binds tightly and sequencespecifically to the promoter. In so doing, MerR inhibits binding to the promoter by RNA polymerase. When $\mathrm{Hg}^{2+}$ is added at low concentrations, the metal ion binds specifically and with high affinity to the DNA-bound MerR, and causes a DNA conformational change detectable by using other metal reagents as conformational probes. This conformational change now facilitates the binding of RNA polymerase and hence activates expression of the gene family.

What are the structural requirements in the metal-binding site? Certainly one requirement is $\mathrm{Hg}$ (II) specificity, so that other metal ions will not also trigger transcriptional activation. Another is high metal-binding affinity to protect the DNA from direct coordination of the $\mathrm{Hg}(\mathrm{II})$. The MerR protein is dimeric, and contains four cysteine residues per monomer. Site-directed mutagenesis studies ${ }^{91}$ have indicated that three of these four cysteine residues are needed for $\mathrm{Hg}$ (II) binding, and EXAFS studies ${ }^{92}$ have been consistent with tricoordinate ligation, clearly a well-designed system for $\mathrm{Hg}$ (II) specificity. Perhaps even more interesting, the site-directed mutagenesis studies ${ }^{91}$ on heterodimers (a mixture of mutant and wild-type monomers) have indicated that the coordinated $\mathrm{Hg}(\mathrm{II})$ bridges the dimer, ligating two cysteines of one monomer and one cysteine of the other. This scheme may provide the basis also for the kinetic lability needed in a rapidly responsive cellular system.

Model systems are also being constructed to explore metal modulation of DNA binding. One system involves the assembly of two dipeptides linked by a central acyclic metal-binding polyether ligand, with $\mathrm{Fe}(\mathrm{EDTA})^{2-}$ tethered to one end to mark site-specific binding. ${ }^{93}$ In the presence of alkaline earth cations, which induce a conformational change that generates a central macrocycle, the linked peptides become oriented to promote sequence-specific binding in the minor groove. In the absence of the alkaline earth ion, no site-specific binding, or cleavage of DNA, is evident. One might consider this system as a simple, first-order synthetic model for the metalloregulatory proteins.

MerR is clearly only one natural metalloregulatory system. Other metal ions bind regulatory factors to mediate the regulation of gene expression in a metalspecific manner. Two examples include the Fe(II)-binding Fur protein from enteric bacteria $^{94}$ and the copper-binding protein ACE1/CUP2 from $S$. cerevisiae. ${ }^{95}$ Both copper and iron are essential trace elements for which high concentrations are toxic; for nucleic acids this toxicity is certainly the result of redox-mediated 

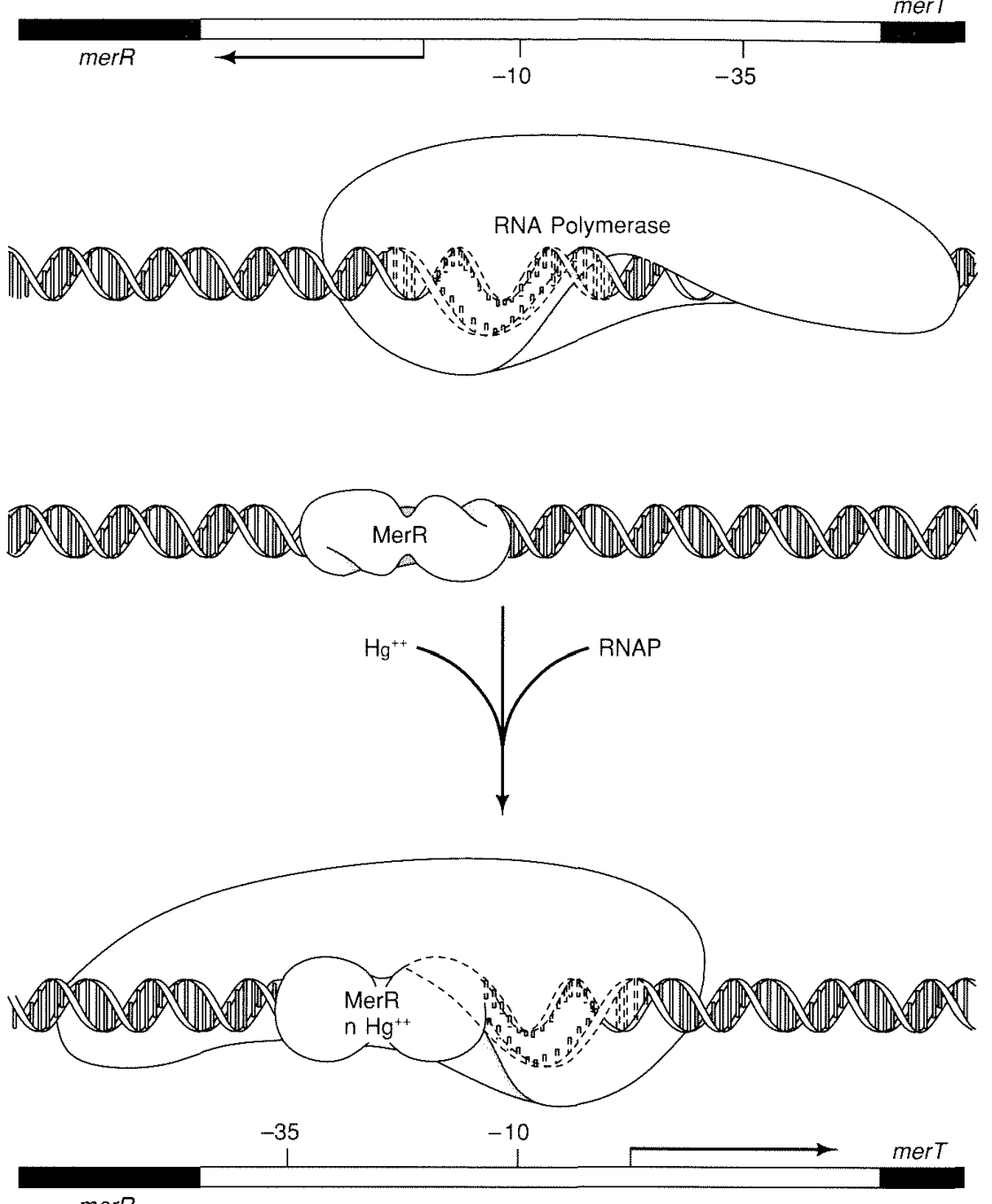

merR

Figure 8.18

A model for MerR metalloregulation. ${ }^{90 a}$ In the absence of MerR, RNA polymerase binds and transcribes the MerR promoter. In the presence of MerR, the preferential binding of MerR to the promoter is observed that inhibits transcription by the polymerase. The addition of $\mathrm{Hg}^{2+}$ then leads to a conformational change that promotes binding of the polymerase, substantially increasing transcription. Reproduced with permission from Reference $90 \mathrm{a}$.

strand damage. Other metal-specific regulatory systems are surely present as well. Both the MerR and the synthetic system may exemplify how these various systems function, how Nature might construct a ligand system to facilitate toxicmetal-specific binding in the presence of DNA that then alters or triggers how other moieties bind and access the nucleic acid. 


\section{A Pharmaceutical Role}

With the exception of cisplatin (see Chapter 9), most pharmaceuticals currently being used as DNA-binding agents were first isolated as natural products from bacteria, fungi, plants, or other organisms. For the most part they represent complex organic moieties, including peptide and/or saccharide functionalities, and often a unique functionality, such as the ene-diyne in calichimycin. These natural products bind DNA quite avidly, through intercalation, groove binding, or a mixture thereof. Often the efficacy of these antitumor antibiotics stems from subsequent alkylation or DNA strand-cleavage reactions that damage the DNA.

Among the various natural products used clinically as antitumor antibiotics are bleomycins, a family of glycopeptide-derived species isolated from cultures of Streptomyces. ${ }^{25,96}$ The structure of bleomycin $\mathrm{A}_{2}$ is shown schematically in Figure 8.19. The molecular mode of action of these species clearly involves binding to DNA and the promotion of single-stranded cleavage at GT and GC sequences. Importantly, as demonstrated by Horwitz, Peisach, and coworkers, this DNA cleavage requires the presence of $\mathrm{Fe}$ (II) and oxygen. ${ }^{97}$ Thus, one might consider Fe-bleomycin as a naturally occurring inorganic pharmaceutical.

What is the role of the metal ion in these reactions? As one might imagine, based upon our earlier discussions of metal-promoted DNA cleavage, the iron center is essential for the oxidative cleavage of the strand through reaction with the sugar moiety. The reaction of Fe(II)-bleomycin can, however, clearly be distinguished from the $\mathrm{Fe}(\mathrm{EDTA})^{2-}$ reactions discussed earlier in that here no diffusible intermediate appears to be involved. Instead of generating hydroxyl radicals, the $\mathrm{Fe}$ center must be positioned near the sugar-phosphate backbone and activated in some fashion to promote strand scission directly.

Despite extensive studies, in fact little is known about how Fe(II)-bleomycin is oriented on the DNA. Indeed, the coordination about the metal is the subject of some debate. The structure of $\mathrm{Cu}(\mathrm{II})-\mathrm{P}-3 \mathrm{~A},{ }^{98}$ a metallobleomycin derivative, is also shown in Figure 8.19. On the basis of this structure and substantive spectroscopic studies on Fe-bleomycin itself, it is likely that, as with $\mathrm{Cu}(\mathrm{II})$, in the $\mathrm{Fe}$ (II)-bleomycin complex the metal coordinates the $\beta$-hydroxyimidazole nitrogen, the secondary amine of $\beta$-aminoalanine, and the N1 nitrogen of the pyrimidine. Whether in addition the primary amines of the amino alanine and of the histidine coordinate the metal is still not settled. Possibly bithiazole coordination or some coordination of the sugar moieties is involved. Nonetheless, given five different coordination sites to the bleomycin, the sixth axial site is available for direct coordination of dioxygen. How is this $\mathrm{Fe}-\mathrm{O}_{2}$ complex oriented on the DNA? It is likely that at least in part the complex binds against the minor groove of the helix. There is some evidence that suggests that the bithiazole moiety intercalates in the helix. It is now becoming clear, however, that the structure of the metal complex itself, its three-dimensional shape, rather than simply the tethered bithiazole or saccharide, is needed for the sequence selectivity associated with its mode of action. 
Although the coordination and orientation of the metal complex are still not understood, extensive studies have been conducted concerning the remarkable chemistry of this species. ${ }^{96,99}$ The overall mechanism of action is described in Figure 8.19. In the presence of oxygen, the $\mathrm{Fe}$ (II) $\mathrm{O}_{2}$ species is formed and is likely rapidly converted to a ferric superoxide species. The one-electron reduction of this species, using either an organic reductant or another equivalent of $\mathrm{Fe}$ (II)-bleomycin, leads formally to an Fe(III)-peroxide, which then undergoes $\mathrm{O}-\mathrm{O}$ bond scission to form what has been termed "activated bleomycin." This species might be best described as $\mathrm{Fe}(\mathrm{V})=\mathrm{O}$ (or $[\mathrm{Fe} \mathrm{O}]^{3+}$ ). This species is comparable in many respects to activated cytochrome $\mathrm{P}-450$ or perhaps even more closely to the Fe center in chloroperoxidase (see Chapter 5). Like these systems, activated bleomycin can also epoxidize olefins and can generally function as an oxo transferase. In contrast to these systems, Fe-bleomycin clearly lacks a heme. How this species can easily shuttle electrons in and out, forming and reacting through a high-valent intermediate, without either the porphyrin sink or another metal linked in some fashion, is difficult to understand. In fact, understanding this process, even independently of our fascination with how the reaction is exploited on a DNA helix, forms the focus of a substantial effort of bioinorganic chemists today.

What has been elucidated in great detail is the reaction of activated bleomycin with DNA. It has been established that the activated species promotes hydrogen abstraction of the $\mathrm{C}^{\prime}-\mathrm{H}$ atom, which is positioned in the minor groove of the helix (Figure 8.19). Addition of another equivalent of dioxgen to this $\mathrm{C} 4$ '-radical leads to degradation of the sugar to form a $5^{\prime}$-phosphate, a 3 '-phosphoglycolate, and free base propenal. Alternatively, oxidation of the $\mathrm{C} 4{ }^{\prime}$-radical followed by hydroxylation in the absence of oxygen yields, after treatment with base, a $5^{\prime}$-phosphate, an oxidized sugar phosphate, and free base.

Other metals such as copper and cobalt can also activate bleomycins, although their mechanistic pathways for strand scission are clearly different from that of $\mathrm{Fe}$ (II)-bleomycin. Whether other natural products that bind DNA also chelate metal ions and exploit them for oxidative strand cleavage is not known, but several systems provide hints that they do. Furthermore, such a fact would not be surprising given our understanding of the utility of metal ions in promoting this chemistry. An even more detailed understanding of this chemistry might lead to the development of second-generation synthetic transition-metal pharmaceuticals that specifically and efficiently target and cleave DNA sites.

\section{A Catalytic Role}

In addition to serving structural and modulating roles in proteins which bind nucleic acids, metal ions also appear to be essential to the functioning of various complex enzymes that act on nucleic acids. At this stage our understanding of the participation of the metal ion in the catalytic chemistry of these enzymes is somewhat sketchy, and we are relying more on our current understanding of the 
(A)

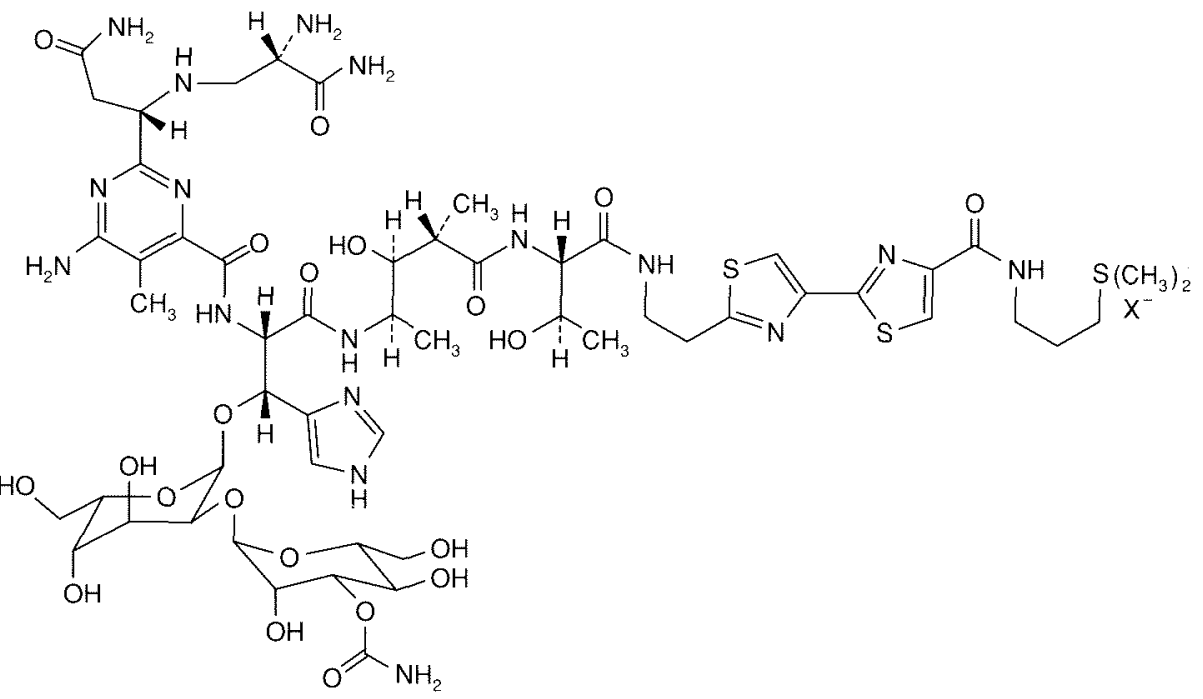

bleomycin

(B)

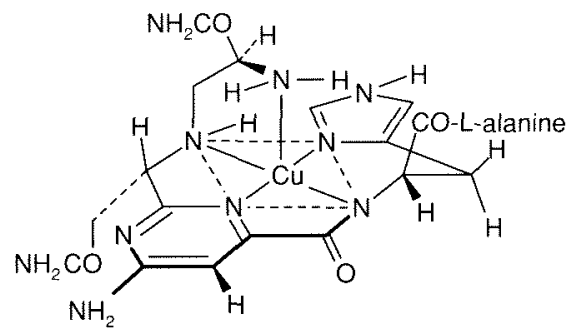

(C)

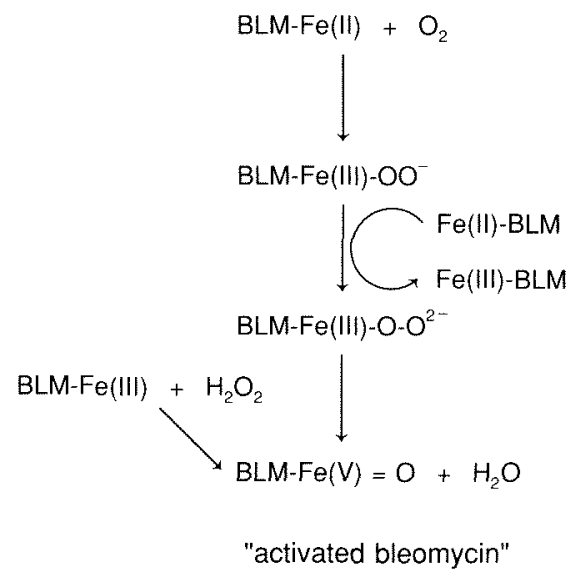


(D)
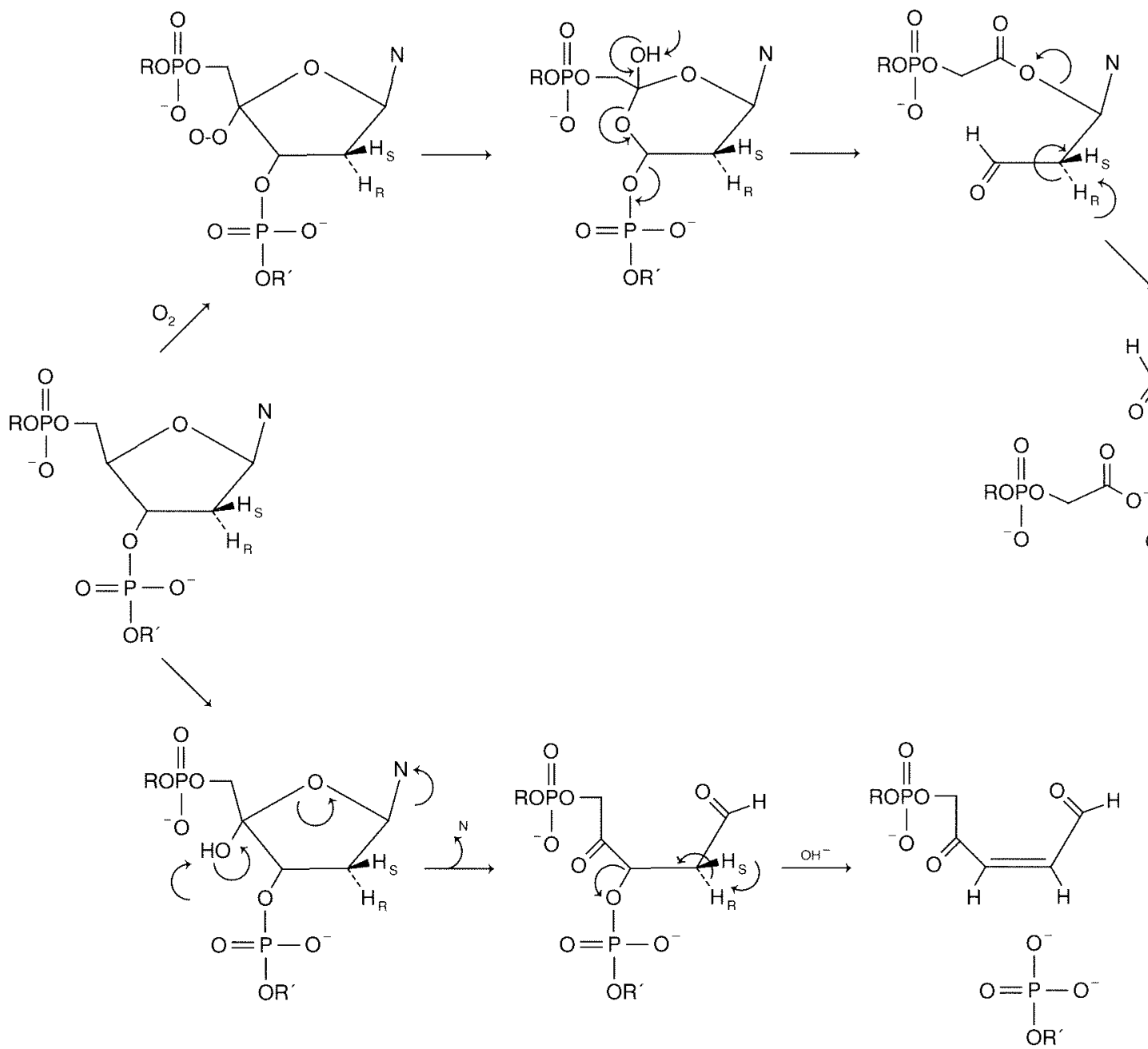

Figure 8.19

(A) The structure of bleomycin $\mathrm{A}_{2}$. (B) The crystallographically determined structure ${ }^{98}$ of a copper derivative of bleomycin, $\mathrm{P} 3 \mathrm{~A}$. (C) A scheme to generate "activated bleomycin." (D) The proposed mechanism of action of activated Fe-bleomycin. 
possible roles where metal ions may prove advantageous. These remain areas of biochemical focus where the inorganic chemist could make a major contribution.

For example, zinc ion appears to be essential to the functioning of both RNA polymerases and DNA topoisomerases. ${ }^{100-102}$ These multisubunit enzymes perform quite complex tasks. RNA polymerase must bind site-specifically to its DNA template, bind its nucleotide and primer substrates, and form a new phosphodiester bond in elongating the growing RNA. Two zinc ions appear to be involved. One may be involved in orienting the nucleotide substrate, and the other structurally in template recognition. It would not be surprising, however-indeed, it might be advantageous-if one or both metal ions also participated in the polymerization step. Our mechanistic understanding of how topoisomerases function is even more cursory. These complex enzymes bind supercoiled DNA, sequentially break one strand through hydrolytic chemistry, move the strand around the other (releasing one tertiary turn), and religate the strand. Again, the zinc ion might participate in the hydrolytic chemistry, the ligation step, or both; alternatively, the metal might again serve a structural role in recognition of the site of reaction.

We do have some understanding of the role of metal ions in several endonucleases and exonucleases. As discussed in Section II.C, metal ions may effectively promote phosphodiester hydrolysis either by serving as a Lewis acid or by delivering a coordinated nucleophile. Staphylococcal nuclease ${ }^{103}$ is an extracellular nuclease of Staphylococcus aureus that can hydrolyze both DNA and RNA in the presence of $\mathrm{Ca}^{2+}$. The preference of the enzyme is for singlestranded DNA, in which it attacks the 5'-position of the phosphodiester linkage, cleaving the $5^{\prime}$-P-O bond to yield a $5^{\prime}$-hydroxyl and $3^{\prime}$-phosphate terminus. $\mathrm{Ca}^{2+}$ ions are added as cofactors and are strictly required for activity. The structure of staphylococcal nuclease, determined by $\mathrm{x}$-ray crystallography and crystallized in the presence of $\mathrm{Ca}^{2+}$ and the enzyme competitive inhibitor pdTp, as well as subsequent NMR and EPR studies on mutant enzymes using $\mathrm{Mn}^{2+}$ as a substitute for the $\mathrm{Ca}^{2+}$ ion, have provided the basis for a detailed structural analysis of the mechanism of this enzyme. In this phosphodiester hydrolysis, the metal ion appears to function primarily as an electrophilic catalyst, polarizing the $\mathrm{P}-\mathrm{O}$ bond, and stabilizing through its positive charge the evolving negative charge on the phosphorus in the transition state. The base is thought here not to be directly coordinated to the metal; instead, action of a general base is invoked.

Metal ions also participate in the functioning of other nucleases, although the structural details of their participation are not nearly as established as those for staphylococcal nuclease. DNAse I also requires $\mathrm{Ca}^{2+}$ for its catalytic activity. ${ }^{104} \mathrm{~S} 1$ endonuclease, mung bean nuclease, and Physarum polycephalem nuclease require zinc ion either as cofactors or intrinsically for nuclease activity, and the restriction enzyme EcoRI may also require intrinsically bound zinc ion. ${ }^{33}$ In terms of how the zinc ion might function in these enzymes, one can look both to staphylococcal nuclease and to bacterial alkaline phosphatase ${ }^{105}$ for some 
illustrations. One would expect that this metal ion could serve both as an electrophilic catalyst and also in the delivery of a zinc-coordinated hydroxide, as it does in alkaline phosphatase, directly attacking the phosphate ester. More work needs to be done to establish the mechanisms by which zinc ion promotes phosphodiester hydrolysis in these enzymatic systems.

Probably most intriguing and mysterious at this stage is the metal participation in the very complex DNA-repair enzyme endonuclease III from E. coli (similar enzymes have also been isolated from eukaryotic sources). This enzyme is involved in the repair of DNA damaged by oxidizing agents and UV irradiation, and acts through an $\mathrm{N}$-glycosylase activity to remove the damaged base, and through an apurinic/apyrimidinic endonuclease activity to cleave the phosphodiester bond adjacent to the damaged site. Although more complex in terms of recognition characteristics, this enzyme functions in hydrolyzing the DNA phosphodiester backbone. What is so intriguing about this enzyme is that it contains a $4 \mathrm{Fe}-4 \mathrm{~S}$ cluster (see Chapter 7) that is essential for its activity! ${ }^{106} \mathrm{We}$ think generally that $\mathrm{Fe}$-S clusters best serve as electron-transfer agents. In the context of this repair enzyme, the cluster may be carrying out both an oxidation and a reduction, to effect hydrolysis, or alternatively perhaps a completely new function for this metal cluster will emerge. (Fe-S clusters may represent yet another structural motif for DNA-binding proteins and one which has the potential for regulation by iron concentration.) Currently the basic biochemical and spectroscopic characterization of the enzyme is being carried out. Understanding this very novel interaction of a metal center and nucleic acid will require some new ideas, and certainly represents one new challenge for the bioinorganic chemist.

\section{REFERENCES}

1. W. Saenger, Principles of Nucleic Acid Structure, Springer-Verlag, 1984; J. K. Barton, Chem. Eng. News 66 (Sept. 26, 1988), 30.

2. M. McCall, T. Brown, and O. Kennard, J. Mol. Biol. 183 (1985), 385.

3. R. Wing et al., Nature 287 (1980), 755.

4. A. H.-J. Wang et al., Nature 282 (1979), 680.

5. S. H. Kim et al., Proc. Natl. Acad. Sci. USA 71 (1974), 4970.

6. E. N. Trifonov and J. L. Sussman, Proc. Natl. Acad. Sci USA 77 (1980), 3816; J. C. Marini et al., Proc. Natl. Acad. Sci. USA 79 (1982), 7664; H.-S. Koo, H.-M. Wu, and D. M. Crothers, Nature 320 (1986), 501 .

7. M. Gellert et al., Cold Spring Harbor Symps. Quant. Biol. 43 (1979), 35; D. M. J. Lilley, Proc. Natl. Acad. Sci. USA 77 (1980), 6468.

8. J. S. Lee et al., Nucleic Acids Res. 12 (1984), 6603; V. I. Lyamichev, J. Biomol. Struct. Dyn. 3 (1986), 667; H. Hun and J. E. Dahlberg, Science 241 (1988), 1791.

9. R. D. Kornberg, Annu. Rev. Biochem. 46 (1977), 931; A. Klug et al., Nature 287 (1980), 509.

10. T. R. Cech, Science 236 (1987), 1532.

11. (a) J. K. Barton and S. I. Lippard, Metal Ions in Biol. 1 (1980), 31; (b) A. M. Pyle and J. K. Barton, Prog. Inorg. Chem. 38 (1990), 413; (c) C. S. Chow and J. K. Barton, Meth. Enzym. 212 (1992), 219.

12. S. E. Sherman et al., Science 230 (1985), 412.

13. D. Hodgson, Prog. Inorg. Chem. 23 (1977), 211.

14. G. L. Eichhorn and Y. A. Shin, J. Am. Chem. Soc. 90 (1968), 7323.

15. L. G. Marzilli, Prog. Inorg. Chem. 23 (1977), 255. 
16. C. H. Chang, M. Beer, and L. G. Marzilli, Biochemistry 16 (1977), 33; G. C. Glikin et al., Nucleic Acids Res. 12 (1984), 1725.

17. M. B. Fleisher, H. Y. Mei, and J. K. Barton, Nucleic Acids and Mol. Biol. 2 (1988), 65.

18. S. J. Lippard, Acc. Chem. Res. 11 (1978), 211.

19. R. V. Gessner et al., Biochemistry 24 (1985), 237.

20. (a) K. W. Jennette et al., Proc. Natl. Acad. Sci. USA 71 (1974), 3839; (b) A. H. Wang et al., Nature 276 (1978), 471.

21. H. Sigel, in T. D. Tullius, ed., Metal-DNA Chemistry, ACS Symposium 402 (1989), 159.

22. R. P. Hertzberg and P. B. Dervan, J. Am. Chem. Soc. 104 (1982), 313; R. P. Hertzberg and P. B. Dervan, Biochemistry 23 (1984), 3934.

23. J. A. Latham and T. R. Cech, Science 245 (1989), 276.

24. P. B. Dervan, Science 232 (1986), 464.

25. S. M. Hecht, ed., Bleomycin, Springer-Verlag, 1979.

26. D. S. Sigman, Acc. Chem. Res. 19 (1986), 180; S. Goldstein and G. Czapski, J. Am. Chem. Soc. 108 (1986), 2244.

27. For other examples of metal-mediated redox cleavage of DNA, see also: N. Grover and H. H. Thorp, J. Am. Chem. Soc. 113 (1991), 7030; X. Chen, S. E. Rokita, and C. J. Burrows, J. Am. Chem. Soc. 113 (1991), 5884.

28. H. Y. Mei and J. K. Barton, J. Am. Chem. Soc. 108 (1986), 7414.

29. H. Y. Mei and J. K. Barton, Proc. Natl. Acad. Sci. USA 85 (1988), 1339.

30. A. M. Pyle, E. C. Long, and J. K. Barton, J. Am. Chem. Soc. 111 (1989), 4520.

31. A. Sitlani et al., J. Am. Chem. Soc. 114 (1992), 2303.

32. M. D. Purugganan et al., Science 241 (1988), 1645.

33. L. A. Basile and J. K. Barton, Metal Ions Biol. Syst., 25 (1989), 31; J. K. Barton, in Frontiers of Chemistry: Biotechnology, Chem. Abstr. Serv., 5 (1989).

34. D. R. Jones, L. F. Lindoy, and A. M. Sargeson, J. Am. Chem. Soc. 106 (1984), 7807.

35. S. H. Gellman, R. Petter, and R. Breslow, J. Am. Chem. Soc. 108 (1986), 2388; J. Chin and X. Zhou, J. Am. Chem. Soc. 110 (1988), 223; J. R. Morrow and W. C. Trogler, Inorg. Chem. 27 (1988), 3387.

36. L. A. Basile, A. L. Raphael, and J. K. Barton, J. Am. Chem. Soc. 109 (1987), 7550.

37. G. L. Eichhorn and Y. A. Shin, J. Am. Chem. Soc. 90 (1968), 7322.

38. R. S. Brown, J. C. Dewan, and A. Klug, Biochemistry 24 (1985), 4785.

39. L. Behlen et al., Biochemistry 29 (1990), 2515.

40. J. K. Barton, Science 233 (1986), 727.

41. J. K. Barton et al., J. Am. Chem. Soc. 108 (1986), 2081.

42. A. M. Pyle et al., J. Am. Chem. Soc. 111 (1989), 3051.

43. C. V. Kumar, J. K. Barton, and N. J. Turro, J. Am. Chem. Soc. 107 (1985), 5518.

44. S. J. Lippard et al., Science 194 (1976), 726.

45. J. K. Barton, J. J. Dannenberg, and A. L. Raphael, J. Am. Chem. Soc. 104 (1982), 4967.

46. J. K. Barton, A. T. Danishefsky, and J. M. Goldberg, J. Am. Chem. Soc. 106 (1984), 2172.

47. R. F. Pasternack, E. J. Gibbs, and J. J. Villafranca, Biochemistry 22 (1983), 2406; R. F. Pasternack and E. J. Gibbs in T. D. Tullius, ed., Metal-DNA Chemistry, ACS Symposium 402 (1989), 59.

48. J. K. Barton and E. Lolis, J. Am. Chem. Soc. 107 (1985), 708.

49. R. E. Mahnken et al., Photochem. Photobiol. 49 (1989), 519.

50. J. P. Rehmann and J. K. Barton, Biochemistry 29 (1990), 1701.

51. J. C. Caradonna et al., J. Am. Chem. Soc. 104 (1982), 5793.

52. J. P. Rehmann and J. K. Barton, Biochemisty 29 (1990), 1710.

53. A. Jack et al, J. Mol. Biol. 111 (1977), 315.

54. M. T. Carter and A. J. Bard, J. Am. Chem. Soc. 109 (1987), 7528.

55. J. K. Barton and A. L. Raphael, J. Am. Chem. Soc. 106 (1984), 2466.

56. M. B. Fleisher et al., Inorg. Chem. 25 (1986), 3549.

57. M. W. van Dyke, R. P. Hertzberg, and P. B. Dervan, Proc. Natl. Acad. Sci. USA 79 (1982), 5470; M. W. van Dyke and P. B. Dervan, Nucleic Acids Res. 11 (1983), 5555.

58. J. K. Barton et al., Proc. Natl. Acad. Sci. USA 81 (1984), 1961; A. E. Friedman et al., Nucleic Acids. Res. 19 (1991), 2595.

59. A. E. Friedman et al., J. Am. Chem. Soc. 112 (1990), 4960; R. Hartshorn and J. K. Barton, J. Am. Chem. Soc. 114 (1992), 5919.

60. W. deHorrocks and S. Klakamp, Biopolymers 30 (1990), 33.

61. R. Tamilarasan, S. Ropertz, and D. R. McMillin, Inorg. Chem. 27 (1988), 4082. 
62. D. J. Galas and A. Schmitz, Nucleic Acids Res. 5 (1978), 3157.

63. T. D. Tullius et al., Methods in Enzym. 155 (1987), 537.

64. R. Law et al., Proc. Natl. Acad. Sci. USA 84 (1987), 9160; C. L. Peterson and K. L. Calane, Mol. Cell Biol. 7 (1987), 4194.

65. J. C. Dabrowiak, B. Ward, and J. Goodisman, Biochemistry 28 (1989), 3314.

66. P. E. Nielsen, C. Jeppesen, and O. Buchardt, FEBS Lett. 235 (1988), 122; C. Jeppesen and P. E. Nielsen, Nucleic Acids Res. 17 (1989), 4947.

67. K. Uchida et al., Nucleic Acids Res. 17 (1989), 10259.

68. A. M. Burkhoff and T. D. Tullius, Cell 48 (1987), 935; A. M. Burkhoff and T. D. Tullius, Nature 331 (1988), 455.

69. B. H. Johnston and A. Rich, Cell 42 (1985), 713; E. Palacek, E. Rasovka, and P. Boublikova, Biochem. Biophys. Res. Comm. 150 (1988), 731.

70. J. K. Barton and A. L. Raphael, Proc. Natl. Acad. Sci. USA 82 (1985), 6460.

71. B. C. Muller, A. L. Raphael, and J. K. Barton, Proc. Natl. Acad. Sci. USA 84 (1987), 1764; I. Lee and J. K. Barton, Biochemistry 32 (1993), 6121.

72. M. R. Kirshenbaum, R. Tribolet, and J. K. Barton, Nucleic Acids Res. 16 (1988), 7948.

73. A. M. Pyle, T. Morii, and J. K. Barton, J. Am. Chem. Soc. 112 (1990), 9432.

74. J. M. Kean, S. A. White, and D. E. Draper, Biochemistry 24 (1985), 5062.

75. G. J. Murakawa et al., Nucleic Acids Res. 17 (1989), 5361

76. C. S. Chow and J. K. Barton, J. Am. Chem. Soc. 112 (1990), 2839; C. S. Chow et al., Biochemistry 31 (1992), 972.

77. H. E. Moser and P. B. Dervan, Science 238 (1987), 645.

78. C. B. Chen and D. S. Sigman, Science 237 (1987), 1197.

79. J. P. Sluka et al., Science 238 (1987), 1129.

80. D. P. Mack, B. L. Iverson, and P. B. Dervan, J. Am. Chem. Soc. 110 (1988), 7572.

81. J. S. Heras et al., J. Biol. Chem. 258 (1983), 14120.

82. J. Miller, A. D. McLachlan, and A. Klug, EMBO 4 (1985), 1609.

83. J. M. Berg, Science 232 (1986), 485.

84. A. Klug and D. Rhodes, Trends Biochem. Sci. 12 (1987), 464; R. M. Evans and S. M. Hollenberg, Cell 52 (1988), 1.

85. J. M. Berg, Proc. Natl. Acad. Sci, USA 85 (1988), 99.

86. M. S. Lee et al., Science 245 (1989), 635; G. Parraga et al., Science 241 (1988), 1489.

87. N. P. Pavletich and C. O. Pabo, Science 252 (1991), 809.

88. (a) T. Pan and J. E. Coleman, Proc. Natl. Acad. Sci. USA 86 (1989), 3145; (b) R. Marmorstein et al., Nature 356 (1992), 408.

89. B. F. Luisi et al., Nature 352 (1991), 497.

90. (a) T. V. O'Halloran, Metal Ions Biol. Syst. 25 (1989), 105; (b) C. T. Walsh et al., FASEB 2 (1988), 124; T. V. O'Halloran and C. T. Walsh, Science 235 (1987), 211.

91. J. D. Helmann, B. T. Ballard, and C. T. Walsh, Science 248 (1990), 946.

92. J. E. Penner-Hahn et al., Physica $B 158$ (1989), 117.

93. J. H. Griffin and P. B. Dervan, J. Am. Chem. Soc. 109 (1987), 6840.

94. A. Bagg and J. B. Neilands, Microbiol. Rev. 51 (1987), 509.

95. P. Furst et al., Cell 55 (1988), 705; C. Buchman et al., Mol. Cell Biol. 9 (1989).

96. J. Stubbe and J. W. Kozarich, Chem. Rev. 87 (1987), 1107.

97. E. A. Sausville, J. Peisach, and S. B. Horwitz, Biochem. Biophys. Res. Comm. 73 (1976), 814.

98. Y. litaka et al., J. Antibiot. 31 (1978), 1070.

99. S. M. Hecht, Acc. Chem. Res. 19 (1986), 383.

100. C. W. Wu, F. Y. Wu, and D. C. Speckhard, Biochemistry 16 (1977), 5449.

101. D. P. Giedroc and J. E. Coleman, Biochemistry 25 (1986), 4946.

102. J. E. Coleman and D. P. Giedroc, Metal Ions Biol. Syst. 25 (1989), 171.

103. A. S. Mildvan and E. H. Serpersu, Metal Ions Biol. Syst. 25 (1989), 309.

104. D. Suck and C. Oefner, Nature 321 (1986), 620.

105. J. E. Coleman, Metal lons in Biol. 5 (1983), 219.

106. H. Asahara et al., Biochemistry 28 (1989), 4444.

107. I am grateful to my students and coworkers for their scientific contributions to some of the work described in this chapter and for their critical review of the manuscript. I also thank in particular Dr. Sheila David for preparation of the figures. 